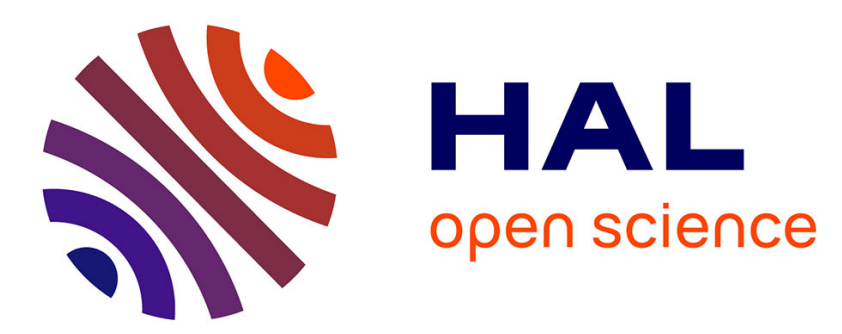

\title{
Les dépressions fermées du Languedoc central et du Roussillon : inventaire, caractérisation géomorphométrique et essai de typologie
}

Mélodie David, Jean-Michel Carozza

\section{- To cite this version:}

Mélodie David, Jean-Michel Carozza. Les dépressions fermées du Languedoc central et du Roussillon: inventaire, caractérisation géomorphométrique et essai de typologie. Géomorphologie: relief, processus, environnement, 2013, Systèmes géomorphologiques: renouveler les thématiques, les outils et les méthodes, 19 (4), pp.407-424. 10.4000/geomorphologie.10376 . hal-02562645

\section{HAL Id: hal-02562645 \\ https://hal.science/hal-02562645}

Submitted on 4 May 2020

HAL is a multi-disciplinary open access archive for the deposit and dissemination of scientific research documents, whether they are published or not. The documents may come from teaching and research institutions in France or abroad, or from public or private research centers.
L'archive ouverte pluridisciplinaire HAL, est destinée au dépôt et à la diffusion de documents scientifiques de niveau recherche, publiés ou non, émanant des établissements d'enseignement et de recherche français ou étrangers, des laboratoires publics ou privés. 


\section{Géomorphologie : relief, processus,}

environnement

vol. 19 - $n^{\circ} 4 \mid 2013$

Systèmes géomorphologiques : renouveler les thématiques, les outils et les méthodes

\section{Les dépressions fermées du Languedoc central et du Roussillon : inventaire, caractérisation géomorphométrique et essai de typologie}

Endorheic depressions of central Languedoc and Roussillon: inventory, geomorphometric approach and typology

Mélodie David et Jean-Michel Carozza

\section{OpenEdition}

\section{Journals}

Édition électronique

URL : http://journals.openedition.org/geomorphologie/10376

DOI : 10.4000/geomorphologie.10376

ISSN : 1957-777X

Éditeur

Groupe français de géomorphologie

Édition imprimée

Date de publication : 15 décembre 2013

Pagination : 407-424

ISBN : 978-2-913282-62-9

ISSN : $1266-5304$

Référence électronique

Mélodie David et Jean-Michel Carozza, « Les dépressions fermées du Languedoc central et du Roussillon : inventaire, caractérisation géomorphométrique et essai de typologie », Géomorphologie relief, processus, environnement [En ligne], vol. 19 - n 4 | 2013, mis en ligne le 15 décembre 2015, consulté le 03 mai 2019. URL : http://journals.openedition.org/geomorphologie/10376 ; DOI : 10.4000/geomorphologie.10376 


\title{
Les dépressions fermées du Languedoc central et du Roussillon : inventaire, caractérisation géomorphométrique et essai de typologie
}

\section{Endorheic depressions of central Languedoc and Roussillon: inventory, geomorphometric approach and typology}

\author{
Mélodie David*, Jean-Michel Carozza**
}

\begin{abstract}
Résumé
Les dépressions fermées sont des formes topographiques en creux, partiellement ou totalement endoréiques, en eau ou hydromorphes. Ces formes ont été décrites de longue date dans la littérature géomorphologique du pourtour du golfe du Lion jusqu'au piémont de la Montagne Noire. Sur le secteur Languedoc central/Roussillon, elles constituent un ensemble d'objets aux morphologies particulièrement hétérogènes, ce qui semble contradictoire avec l'hypothèse d'un processus de morphogénèse unique. L'objectif de ce travail est donc la caractérisation quantitative de ces formes par une approche géomorphométrique et la réalisation d'une typologie rendant compte de cette diversité. Dans un premier temps, un inventaire exhaustif des dépressions a été entrepris entre le Languedoc central et le Roussillon à partir d'un ensemble de sources cartographiques et toponymiques. 175 dépressions ont été identifiées dans les basses plaines du Roussillon, de l'Aude et de l'Hérault, dont 61 sont inédites. Sur ce corpus, 83 formes ont une taille suffisante pour permettre une étude morphométrique. À partir de sept paramètres descriptifs mesurés sous SIG (lithologie, surface de la dépression sensu largo, surface de la cuvette hydromorphe, surface du bassin-versant drainé, incision de la forme, encaissement de la forme dans le paysage et indice de circularité), un premier essai de typologie est proposé par la réalisation d'une ACP. Il met en évidence l'existence de trois grands groupes de modelés dont l'origine est discutée.
\end{abstract}

Mots clés : Languedoc-Roussillon, modelé hydro-éolien, tectonique salifère, géomorphologie, géomorphométrie, ACP.

\begin{abstract}
Enclosed depressions are hollow landforms, which are partially or totally endorheic. These forms were described in the geomorphological literature, found from the Gulf of Lion to the foothills of the Montagne Noire. In central Languedoc and Roussillon, there is a set of depressions with a heterogeneous morphology, which appear to contradict the theory of a single aeolian morphogenic process. The aim of this study is first to provide a quantitative analysis of these forms using a geomorphometric approach and then to propose a typology. First, a comprehensive inventory of these depressions was undertaken between central Languedoc and Roussillon, from a set of cartographic and toponymic sources. 175 depressions were identified in the lowlands of Roussillon, Aude and Hérault, 61 of which were not investigated. Of the remaining 175, 83 depressions were of sufficient size to be included in the morphometric study. From seven descriptive parameters measured in GIS (lithology, area of the depression sensu largo, area of the hollow wetland, area of the drained catchment, incision, incision of the shape in the landscape and circularity index), a first typology is proposed by performing PCA. It highlights the existence of three major types of landforms for which origin is discussed.
\end{abstract}

Key words: Languedoc-Roussillon, aeolian scouring, salt tectonic, geomorphology, geomorphometry, PCA.

\section{Abridged English version}

Enclosed depressions are hollow landforms, which are naturally endorheic but can become partially exorheic under human actions. This kind of form is widespread around the world and can be the result of various morphogenic processes. In the area of the Gulf of Lion, these forms are numerous and have been studied for a long time. However, morphogenic process(es) that lead to the formation of the depressions are still in discussion. Among the numerous genesis hypotheses proposed, most have been partially refuted because they did not account for all the forms (Gottis, 1968). About twenty years ago, the idea of a single niveo-aeolian morphogenic process in periglacial context was proposed and is now broadly accepted (Ambert and Clauzon, 1992). This hypothesis was first developed to explain the origin of the depres-

* GEODE - UMR 5602 CNRS/UTM (melodie.david@etu.univ-tlse2.fr).

** Faculté de Géographie, Université de Strasbourg et GEODE - UMR 5602 CNRS/UTM (carozza@unistra.fr). 
sions in the Istres area (Ambert, 1973; fig. 1). Later, this model was generalised to include the entire central Languedoc and Roussillon region (fig. 1 and fig. 3) by P. Ambert and G. Clauzon (1992). However, the morphology of enclosed depressions largely varies with regard to shape and area and thus contradicts a single, identic process of formation. In addition, some elements are in contradiction with the very idea of an aeolian action (i.e., attested diapiric origin of the Étang du Doul in Peyriac-de-Mer (fig. 2E, fig. 9, and fig. 10C); abrupt slope facing the dominant wind in some cases; important thickness of deposits at the bottom of some forms).

The aim of this paper is to propose a geomorphometric analysis of enclosed depressions of central Languedoc and Roussillon. This work consists of three main steps: (i) Comprehensive inventory of enclosed depression forms based on previous works (Bécat, 1977; CENLR, 2000; Carozza et al., in press) and completed by new data based on three criteria (geological, toponymic and geomorphological); (ii) A quantitative characterisation of 3-D forms and their diversity according to 7 indicators (fig. 3 and fig. 4): lithology, area of the depression sensu largo $(S, h a)$, area of the hollow wetland (If, \% of the form), area of the drained catchment (Ib,\% of the form), incision (Ii, $\left.\mathrm{mm}^{-2}\right)$, incision of the shape in the landscape (Ie, $\mathrm{mm}^{-2}$ ) and circularity index ( $\mathrm{Kg}$ ); (iii) A typology by performing PCA based on computed geomorphometric indicators. The latter analysis should allow us to propose relationships between quantitative parameters of forms and genetic processes.

175 depressions have been identified in the lowlands of central Languedoc and Roussillon (fig. 5). Among them, 61 forms were previously undocumented in the literature. Due to documentation spatial resolution restrictions (DEM spatial resolution $50 \mathrm{~m}$ and metric precision), only 83 depressions of the inventory have sufficient size to allow delimitation and characterisation with geomorphometric indicators. The univariate geomorphometric analysis (fig. 6) reveals the heterogeneity of the data set. The PCA (fig. 7) then allows us to propose a typology of three groups of depressions (fig. 7 to fig. 10). The first group (g1) includes 5 depressions, characterised by their size, important hollow wetland, and no circularity. The second group (g2) includes 14 depressions, which are small forms with strong incision in the landscape and strong circularity. The last group (g3) comprises the majority (64) of the depressions. Group g3 anti correlates with groups $\mathrm{g} 1$ and $\mathrm{g} 2$ and is characterised by drained catchment area.

Aeolian processes likely created the most extensive and complex forms with a low incision, which are concentrated in the windy corridor of the Aude valley (Tramontane and Cers). They constitute most of the group gl (e.g., Étang de Capestang; fig. 9). Aeolian erosion could also be the cause of the coalescence of small forms which are partially represented in group g3 (e.g., set of Retenue d'eau/Estagnols in Villeneuve-de-la-Raho; fig. 9). The wind could also have formed the small depressions on the Pleistocene terraces, which correspond to the group g2 (e.g., Le Clot à Nizas; fig. 9 and fig. 10B).

Regarding circular depressions, which present abrupt slope facing the dominant wind (e.g., Canohes or Sainte-Croix in
Sigean; fig. 2C, fig. 2D, fig. 9, and fig. 10D), the role of the aeolian erosion in the genesis is more difficult to accept. This kind of form that is in group 3 and in group 1, could be impacted by wind in different ways: (i) a partial rework of the limits of the depression; (ii) a role in flushing out of sediments outside of reservoirs during cold events; (iii) formation of pebble pavement layer dreikanter like shape on quartzitic or marble (e.g., in the depression of Bages; Carozza, in press; fig. 2A and fig. 9). However, as no orientation in the direction of dominant wind is observed for this form, we cannot cite aeolian erosion as the first process of genesis. The latter forms are close to the diapiric shape of the Etang du Doul (fig. 2E, fig. 9 and fig. 10C). If we remain cautionous, the hypothesis of a diapiric origin for some depressions of groups g3 and g1 could be considered. Indeed, many depressions of these groups present salt accumulations, which are reflected by development of haline vegetation and in some cases, salt exploitation during exceptional drought (e.g., depressions of Villeneuve-de-la-Raho; Martzluff, 1993; Montady, Abbé, 2009; fig. 2B, fig. 9, and fig. 10A). Fieldwork is necessary to confirm this last hypothesis.

\section{Introduction}

Très largement répandues à l'échelle du globe, les dépressions fermées sont des formes qui ont été mises en relation avec des processus morphogéniques variés : cratères d'impacts météoriques (Sears, 1978 ; Norton, 1998), cratères d'explosions volcaniques ou volcano-phréatiques (Degeai, 2004), affaissements de buttes cryogènes de type palse ou pingo (Pissart, 1987 ; French et Demitroff, 2001 ; Van Vliet-Lanoë, 2005), dissolution superficielle ou soutirage karstique (Nicod, 1991 ; White and White, 2006 ; Kranjc, 2013), déflation éolienne ou nivo-éolienne (Goudie et Wells, 1995 ; French et Demitroff, 2001) et effondrement de diapir lié à la tectonique salifère (Brun et Fort, 2008 ; Alsop et al., 2012) ou une combinaison d'actions comme la dissolution et de déflation éolienne (Sanchez et al., 1997).

D'un point de vue géomorphologique, les dépressions fermées sont des cuvettes naturellement endoréiques ou partiellement endoréiques (seuils de débordements), de dimension variable (depuis quelques dizaines de $\mathrm{m}^{2}$ à plusieurs $\mathrm{km}^{2}$ ), qui peuvent avoir été rendues exoréiques sous l'action de l'Homme au moins depuis le Moyen Âge (Puig, 2003 ; Abbé, 2006). Elles peuvent constituer de simples cuvettes topographiques sèches ou occupées par des plans d'eau permanents (étangs, lacs), des zones temporairement en eau ou hydromorphes (marais ou prairies humides). Ces formes, d'allure plus ou moins évasée, peuvent être encaissées de quelques décimètres à plusieurs dizaines de mètres. Elles se caractérisent par la juxtaposition de deux unités morphologiques distinctes. La partie basse, qui correspond à la dépression sensu stricto de la forme, est occupée par la cuvette en eau ou hydromorphe, à pente faible. Cette cuvette est entourée par des versants ou des glacis qui présentent une pente plus marquée, qui s'adoucit sur la partie haute de la forme et délimite ainsi le contour de la dépression sensu largo (impluvium). Ces versants peuvent s'ouvrir sur de véritables bassins 
versants plus ou moins vastes, qui contribuent au bilan hydrologique de la dépression (Yechieli et Wood, 2002).

Ce type de forme a été identifié de longue date en zone méditerranéenne, notamment sur le pourtour du golfe du Lion, où il est omniprésent (Gottis, 1968 ; Ambert, 1994). Cependant dans cet environnement, le ou les processus de genèse à l'origine de leur formation reste(nt) discuté(s). Parmi les nombreuses hypothèses morphogénétiques qui ont été proposées, nombre d'entre elles ont été partiellement réfutées car elles ne permettent pas de rendre compte de la diversité des formes (Gottis, 1968). Ainsi, l'hypothèse d'une origine unique apparaît difficile à maintenir. Toutefois, depuis une vingtaine d'années, l'idée d'un façonnement nivéo-éolien en contexte périglaciaire proposé par H. Arnal (1971) pour l'étang de Pujaut a été largement diffusée (Ambert et Clauzon, 1992). Développée d'abord pour expliquer le modelé des dépressions de la région d'Istres (Ambert 1973 ; fig. 1), elle reposait alors sur une série de critères stricts, notamment : 1) une orientation préférentielle des cuvettes dans la direction du vent dominant (le Mistral), souvent en alignements ; 2) une forme elliptique des cuvettes, assimilable à des cupules éoliennes dissymétriques de petite taille à bords parallèles ; 3) la présence de roches favorables à ce type de morphogénèse (principalement les formations de molasse éocène à tertiaire à faible cohésion des matériaux) ; 4) l'affleurement du substratum sous une fine couche de colluvions dans la dépression et 5) une action de la déflation marquée sur les plateaux par des cailloutis dont toute trace de pédogenèse a été balayée, ainsi que par des traces de façonnement éolien des galets (dreikanter). Cette hypothèse d'une origine nivéo-éolienne a ensuite été étendue à la plaine du Roussillon (interfluve Têt / Tech ; fig. 1) et au Languedoc central (région de Capestang / Béziers ; Ambert et Clauzon, 1992 ; fig. 1). Ces régions ont en commun une efficacité avérée de l'érosion éolienne (Tramontane, Cers, rapport in Ambert et Clauzon, 1992) et son exacerbation probable lors des périodes froides du Pléistocène en liaison avec un climat froid et des conditions xériques (Jalut et al., 2009). Ce mécanisme a été généralement admis et généralisé à l'ensemble du golfe du Lion dans la littérature sans réelle revue critique.

Cependant, rares sont les dépressions du Languedoc et du Roussillon qui correspondent au modèle type dé- fini en Pays d'Istres : 1) l'orientation des formes dans le sens du vent dominant (NW) est loin d'être la situation la plus courante ; 2) certaines formes présentent un côté abrupt opposé au vent dominant, qui ne permet pas de les assimiler à des cupules éoliennes de grande taille et rend difficile l'exportation du matériel par le vent et 3) un remblaiement sédimentaire important est observé dans certaines formes qui tempère l'hypothèse d'un balayage efficace des particules. Cependant, celui-ci est généralement tardi- à postglaciaire dans les rares cas où des datations sont disponibles (Guilaine, 1993). L'attribution de l'ensemble des formes au Pléistocène (principalement moyen à supérieur) repose principalement sur : 1) la corrélation avec les formations éoliennes des piémont et massifs voisins (Ambert in Guilaine, 1993) ; 2)

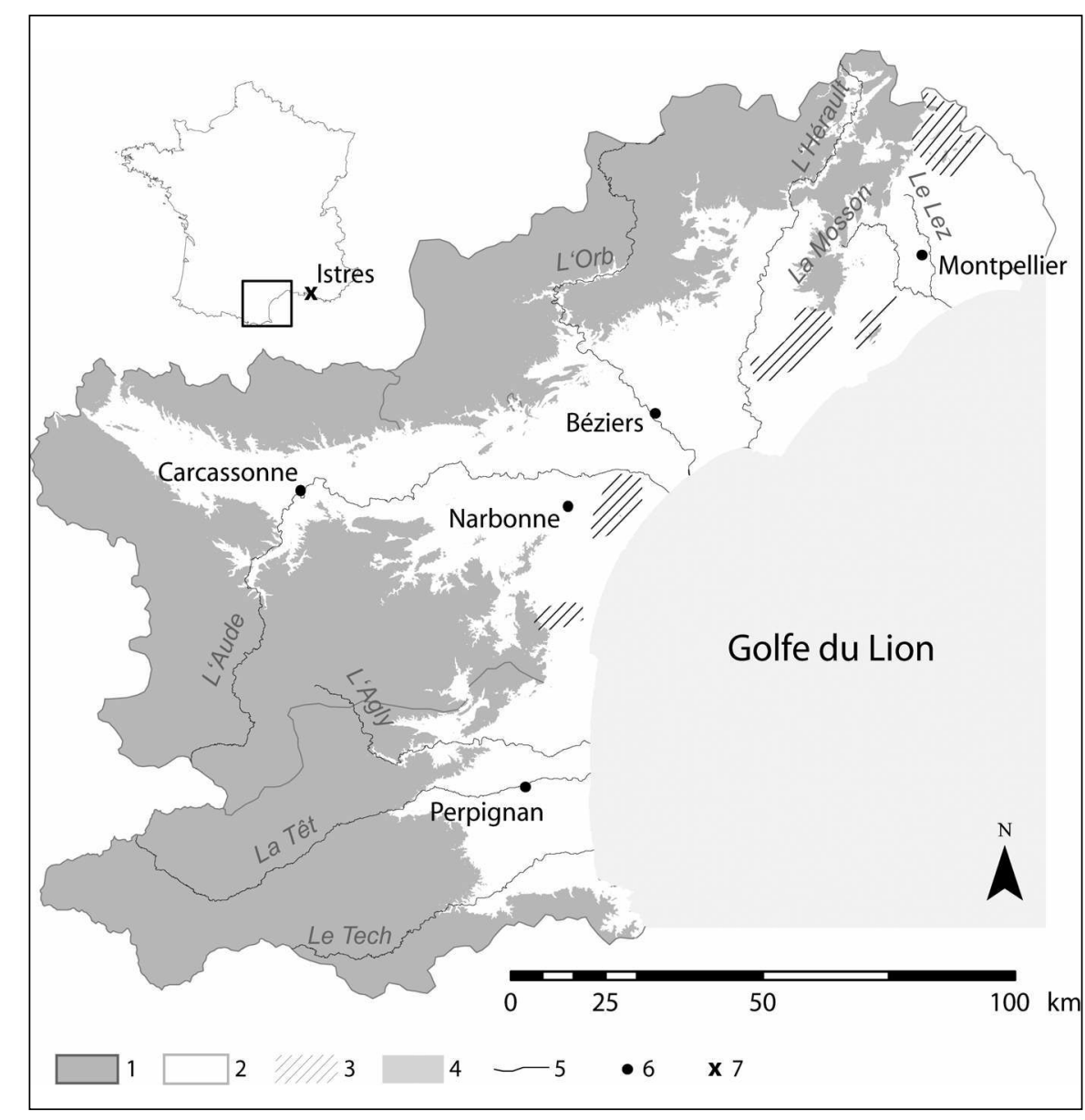

Fig. 1 - Basses plaines du Languedoc central et du Roussillon. 1 : Languedoc central et Roussillon avec, du nord au sud, les départements de l'Hérault, de l'Aude et des PyrénéesOrientales ; 2 : basse plaine (<200 m NGF) ; 3 : secteurs karstiques de la basse plaine avec du nord au sud les Garrigues de Saint-Mathieu-de-Tréviers, la Montagne de la Gardiole, la Garrigue de Villeveyrac, la Montagne de la Clape et la pointe des Corbières ; 4 : Mer Méditerranée ; 5 : éléments principaux du réseau hydrographique ; 6 : principales agglomérations ; 7 : secteur d'Istres, sur lequel est basée l'hypothèse d'un processus de morphogénèse nivéoéolien unique à l'origine des dépressions (Ambert, 1973).

Fig. 1 - Lowlands of central Languedoc and Roussillon. 1: central Languedoc and Roussillon with counties of Hérault, Aude, and Pyrénées-Orientales, from north to south; 2: lowlands (<200 m ASL - depending on Marseille tide gauge); 3: karstic areas in the lowland with Garrigues de Saint-Mathieu-de-Tréviers, Montagne de la Gardiole, Garrigue de Villeveyrac, Montagne de la Clape, and Pointe des Corbières, from north to south; 4: Mediterranean Sea; 5: main components of hydrographical network; 6 : major cities; 7: Istres area where the theory of a single niveo-aeolian morphogenic process in periglacial context was first developed to explain the origin of the depressions (Ambert, 1973). 


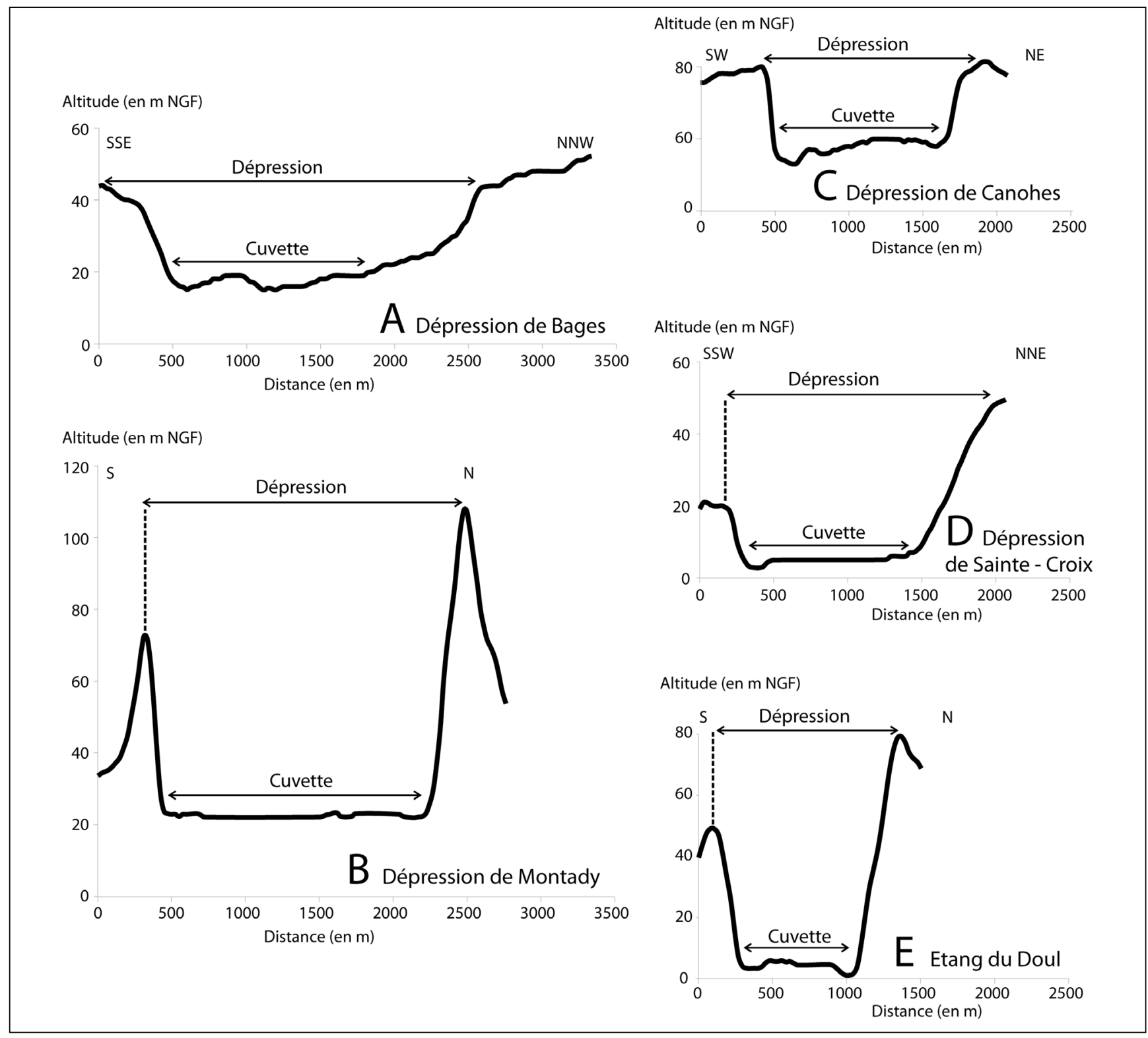

Fig. 2 - Profils topographiques de quelques dépressions du Languedoc central et du Roussillon (Bages, Montady, Canohes, Sainte-Croix à Sigean et l'Etang du Doul à Peyriac-de-mer ; données IGN). Pour chaque dépression le diamètre de la forme (en $\mathrm{m}$ ) apparait en abscisse et l'altitude (en m NGF) en ordonnée. La dépression sensu largo et la cuvette hydromorphe sont respectivement indiquées par une flèche horizontale. Ces formes sont localisées sur la fig. 9.

Fig. 2 - Topographic profiles of some enclosed depressions of central Languedoc and Roussillon (Bages, Montady, Canohes, Sainte-Croix in Sigean, and Etang du Doul in Peyriac-de-mer; data from IGN). For each depression the graph indicates the diameter of the form (in $\mathrm{m}$ ) in abscissa and the elevation in ordinate (in $m$ ASL - depending on Marseille tide gauge). Horizontal arrows show the depression sensu largo (up arrow) and the hollow wetland (down arrow). The location of these depressions is shown in fig. 9.

la datation des remblaiements qui permet l'attribution de l'arrêt du dernier fonctionnement mais qui ne correspond pas nécessairement à l'âge de façonnement de la forme et 3) une chronologie relative par rapport aux niveaux d'édification des terrasses alluviales. De plus, la mise en relation de l'ensemble des dépressions du golfe du Lion avec ce processus unique de façonnement est parfois contredite. Ainsi, dans le cas de la dépression du Doul à Peyriac-de-Mer (secteur de Sigean, fig. 2, fig. 9 et fig. 10), pourtant proche par sa position et sa morphologie des grandes dépressions du Languedoc central et du Roussillon, l'origine diapirique est attestée par la présence de quartz bipyramidaux rouges caractéristiques des niveaux de sel triasique (Barrot, 1975 ; Lespinasse, 1982) liés à la tectonique distensive qui affecte la marge du golfe du Lion du Miocène inférieur au Pliocène (Gaullier, 1993) et à la réactivation de la faille de Portel (Gorini, 1993).

La grande diversité morphologique des dépressions du Languedoc central et du Roussillon est démontrée par les différents travaux qui ont été menés. D'une part, une série de monographies décrit de façon détaillée plusieurs grandes dépressions emblématiques telles que celles de Villeneuve- 
de-la-Raho (Azemar et Carriere, 1980 ; Martzluff, 1993 ; fig. 9), de Montady (Carriere, 1980 ; Abbé, 2009 ; fig. 2, fig. 9 et fig. 10), de Marseillette (Chabert, 1980 ; Guilaine, 1993 ; fig. 9) ou encore de Capestang (Derruau, 1996 ; fig. 9). D'autre part, des travaux d'inventaire départementaux (Bécat, 1977) ou régionaux (CENLR, 2000) et des travaux historiques sur les zones humides (Caucanas, 1995 ; Abbé, 2005), identifient des formes de taille plus réduite et moins marquées dans les paysages, voire totalement effacées.

Le présent travail consiste en l'analyse morphométrique des dépressions fermées du Languedoc central et du Roussillon dans un triple objectif : 1) caractériser d'un point de vue quantitatif les formes des dépressions fermées et leur diversité ; 2) réaliser une typologie de ces formes afin de mettre en évidence des descripteurs pertinents et des parentés ; 3) Proposer des hypothèses de relation entre paramètres quantitatifs des formes et processus morphogéniques afin, le cas échéant, de discriminer des processus responsables de leur genèse. Cette démarche a été appliquée aux dépressions des basses plaines littorales du Languedoc central et du Roussillon. Elle s'appuie sur une révision de l'inventaire des formes, qui permet la constitution d'un corpus le plus exhaustif possible. Sur cette base, une analyse morphométrique et une typologie ont été établies et la relation forme / processus est discutée.

\section{Zone d'étude}

Les basses plaines du Languedoc central/Roussillon forment une frange littorale large d'une dizaine à une trentaine de kilomètres, qui pénètre parfois dans l'arrière pays par l'entaille des principales vallées (fig. 1 et fig. 3) : celles de

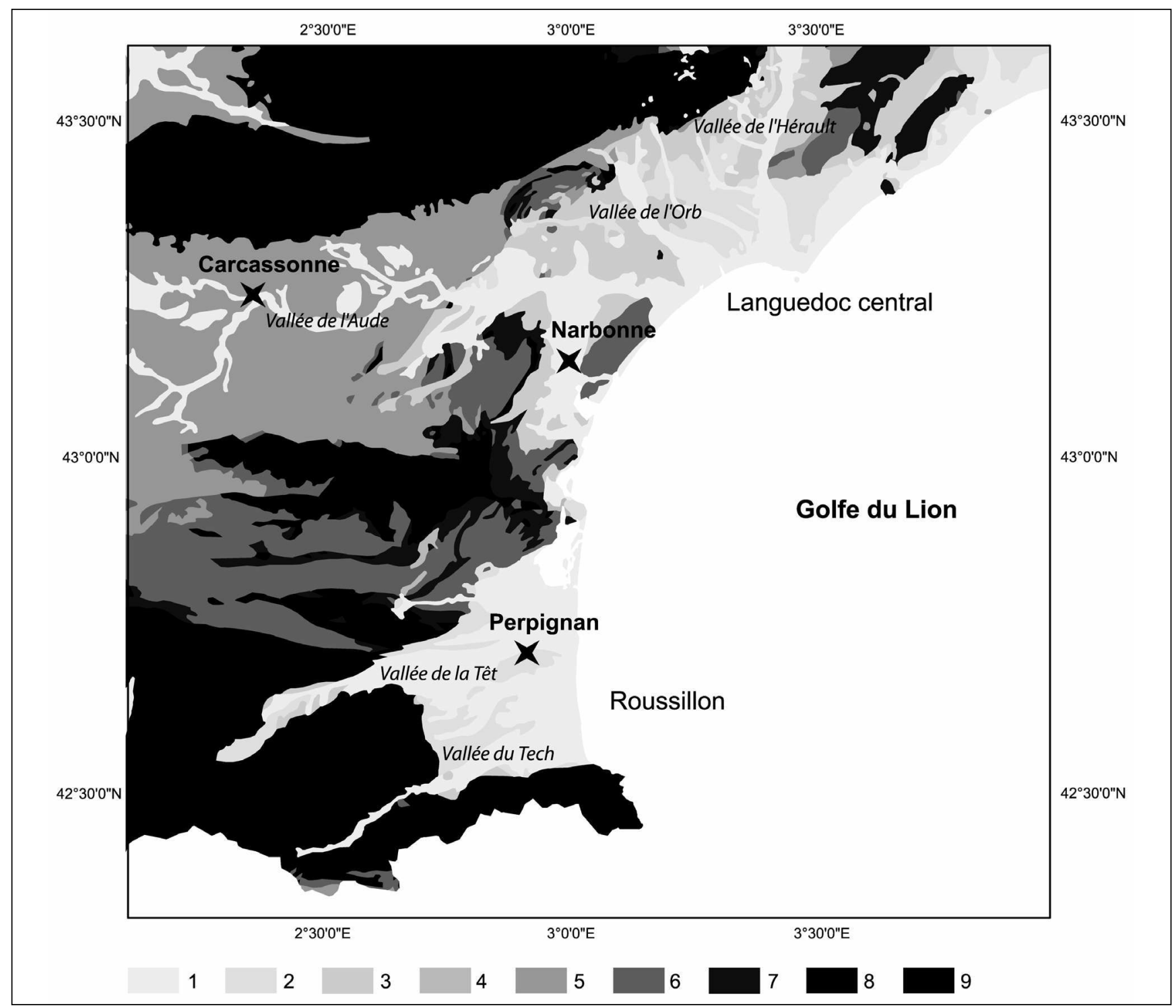

Fig. 3 - Carte géologique simplifiée de la région étudiée (données OneGeology). $1:$ Quaternaire ; $2:$ Pliocène ; $3:$ Miocène ; 4 : Oligocène ; 5 : Eocène ; 6 : Crétacé $; 7$ : Jurassique ; 8 : Trias ; 9 : Paléozoïque et Précambrien.

Fig. 3 - Simplified geological map of the studied area (data from OneGeology). 1: Quaternary; 2: Pliocene; 3: Miocene; 4: Oligocene; 5: Eocene; 6: Cretaceous; 7: Jurassic; 8: Triassic; 9: Paleozoic and Precambrian. 
la Têt, du Tech et de l'Agly, en Roussillon, et celles de l'Aude, de l'Hérault et de l'Orb en Languedoc. Au-delà, se développent les plateaux, causses et arrières pays fréquemment calcaires. La limite entre ces dernières unités et les basses plaines se situe vers $200 \mathrm{~m}$ d'altitude (fig. 1). Ces basses plaines correspondent à une série de blocs et bassins associés à la marge passive occidentale du golfe du Lion, comprise entre les Pyrénées à l'ouest et le delta du Rhône à l'est. Les formations néogènes et quaternaires (fig. 3) couvrent la majeure partie de la zone et constituent un piémont disséqué (Calvet, 1996). Sous cette couverture néogène, diverses formations originales se reconnaissent, notamment des niveaux de gypse miocène, responsables de phénomènes halocinétiques au large de la Méditerranée, qui affleurent par endroit (Le Cann, 1987 ; Seranne, 1999) et les formations de sel triasique partout présentes en profondeur (Lespinasse, 1982). Ces dernières formations, associées à une structure particulièrement faillée consécutive à l'extension mio-pliocène (Seranne et al., 1995), forment un terrain propice au diapirisme.

La basse plaine du Languedoc central/Roussillon peut être subdivisée en trois unités morphologiques/paysagères : le littoral, les deltas holocènes et les piémonts mio-plio-quaternaires. La continuité du piémont néogène est interrompue par des pointements calcaires (Garrigue de Saint-Mathieu de Tréviers, Garrigue de Villeveyrac et pointe du massif des Corbières ; fig. 1). Il en va de même pour la frange littorale (Montagne de la Gardiole et Montagne de la Clape ; fig. 1). Dans ce travail et afin de simplifier le traitement des données, le secteur pris en compte correspond à la frange comprise entre 0 et $200 \mathrm{~m}$ d'altitude, qui délimite grosso modo la transition piémont-massifs calcaires et la zone littorale (fig. 1). Les étangs littoraux et la zone des deltas holocènes ont été exclus de ce travail car leur ennoiement ne permet pas une bonne prise en compte de leurs caractéristiques morphométriques, notamment de la profondeur. À l'intérieur de l'espace de travail, les zones d'affleurement des massifs calcaires mésozoïques n'a pas été prise en considération afin d'éliminer de l'inventaire les formes associées au karst.

\section{Matériels et méthodes}

\section{Données utilisées}

Ce travail se base essentiellement sur une reconnaissance des formes à partir de supports cartographiques. Deux types de sources ont été utilisés. Deux dalles du modèle numérique de terrain (BDAlti) de l'IGN, à une résolution spatiale de $50 \mathrm{~m}$ et de précision métrique, ont permis de couvrir la totalité de la zone d'étude. Cette base de donnée altimétrique était la plus précise au moment de la réalisation de ce travail. Les sources cartographiques conventionnelles ont été également utilisées. Les cartes géologiques du BRGM au 1/50 000 ont permis de reconnaître un certain nombre de formes d'après leur remblaiement fluvio-colluvial généralement attribué à l'Holocène. Dans le cas de l'Est de la plaine du Roussillon, pour laquelle la cartographie du BRGM n'est pas disponible, une cartographie au 1/25000 inédite a été utilisée. La carte topographique de l'IGN au 1/25 000 a éga-
Fig. 4 - Unités morphologiques et indicateurs morphométriques pour la caractérisation des dépressions fermées. Les numérotations de 1 à 6 dans les cercles noirs font le lien entre les parties $\mathrm{A}, \mathrm{B}$ et $\mathrm{C}$ de la figure. $\mathrm{A}$ : Vue en plan. 1 : dépression sensu largo ; 2 : cuvette hydromorphe (pente $<3^{\circ}$ ) ; 3 : bassin-versant ; 4 : buffer de $500 \mathrm{~m}$ autour de la forme ; 5 : point haut de la dépression (altmax) :6: point bas de la dépression (altmin) $; 7:$ point haut de l'environnement (altculm ; pris sur le buffer) ; 8 : isohypse $(2,5 \mathrm{~m})$; 9 : exutoire artificiel. B : Vue en profil. Eléments de la géomorphologie des dépressions fermées caractérisés par les indices morphométriques présentés en $\mathrm{C}$. $\mathrm{C}$ : Détail des indices morphométriques. Le tableau indique (de gauche à droite) : les noms des indices morphométriques; le type de calcul effectué ; le recours ou non à une normalisation; la méthode de calcul (où $f\left(e \mathrm{~m}^{2}\right.$ ) est la surface de la cuvette hydromorphe ; $b\left(\mathrm{en}^{2}\right.$ ) est la surface du bassin versant et $P(e n m)$ est le périmètre de la dépression) et l'objectif visé. 1 : la surface de la dépression sensu largo ( $\mathrm{S}$, en ha) a été mesurée automatiquement sans aucune normalisation. $S$ vise à caractériser la taille de la dépression ; 2 : l'indice de surface de la cuvette hydromorphe (If, en \%) a été calculé automatiquement et normalisé selon la surface de la dépression (S). If vise à caractériser la forme du profil en travers ; 3 : l'indice de surface du bassin-versant (Ib, en \%) a été calculé automatiquement et normalisé selon la surface de la dépression (S). lb vise à caractériser l'étendue du bassin du drainage ; 4 : l'indice d'incision (li, en $\mathrm{mm}^{-2}$ ) a été calculé semi-automatiquement et normalisé selon la surface de la dépression (S). li vise à caractériser la profondeur de la forme ; 5 : l'indice d'encaissement dans le paysage (le, en $\mathrm{mm}^{-2}$ ) a été calculé semi-automatiquement et normalisé selon la surface de la forme (S). le vise à caractériser l'empreinte de la forme dans son environnement ; 6 l'indice de circularité de Gravelius $(\mathrm{Kg})$ a été calculé semi-automatiquement sans aucune normalisation. $\mathrm{Kg}$ vise à caractériser la tendance à la circularité de la forme.

Fig. 4 - Morphological units and morphometric parameters used for the characterisation of endorheic depressions. Black circles with numbers 1 to 6 act as a bridge between parts $A, B$ and $C$ of the figure. A: Plan view. 1: depression sensu largo; 2: hollow wetland (slope $\left\langle 3^{\circ}\right.$ ); 3: catchment; 4: buffer of $500 \mathrm{~m}$ from the depression; 5: highest point of the depression (altmax); 6: lowest point of the depression (altmin); 7: highest point of the environment / buffer (altculm); 8: isohypse (2.5 m); 9: artificial outlet. B: Profile view. Geomorphological features of the depressions related to the morphometric parameters which appear in C. 1: depression sensulargo; 2: hollow wetland; 3: catchment; 4: incision; 5: incision of the shape in the landscape; 6: circularity of the shape. C: Details of the morphometric parameters. The table gives (from left to right): the names of the morphometric parameters; the kind of calculation which were used; details on the normalisation; the calculation method (where $f$ (in $m^{2}$ ) is the area of the hollow wetland, $b$ (in $m^{2}$ ) is the area of the catchment and $P$ (in $m$ ) is the perimeter of the depression) and the kind of characterisation which is expected. 1: area of the depression sensu largo $(S$, in ha) is automatically calculated, with no normalisation. $S$ should characterise the depression size; 2: area of the hollow wetland index (If, in \%) is automatically calculated and is normalised in relation to the area of the depression (S). If should characterise the cross section shape; 3 : area of the catchment index $(\mathrm{Ib}$, in \%) is automatically calculated and is normalised in relation to the area of the depression (S). Ib should characterise the extent of the catchment; 4: incision index (li, in $\mathrm{mm}^{-2}$ ) is partially automatically calculated and is normalised in relation to the area of the depression (S). li should characterise the depression depth; 5: index of shape incision in the landscape (le, in $\mathrm{mm}^{-2}$ ) is partially automatically calculated and is normalised in relation to the area of the depression (S). Ie should characterise the imprint of the depression in the landscape; 6: Gravelius index/circularity index $(\mathrm{Kg})$ is partially automatically calculated, with no normalisation. Kg should characterise the depression tendency to circularity. 


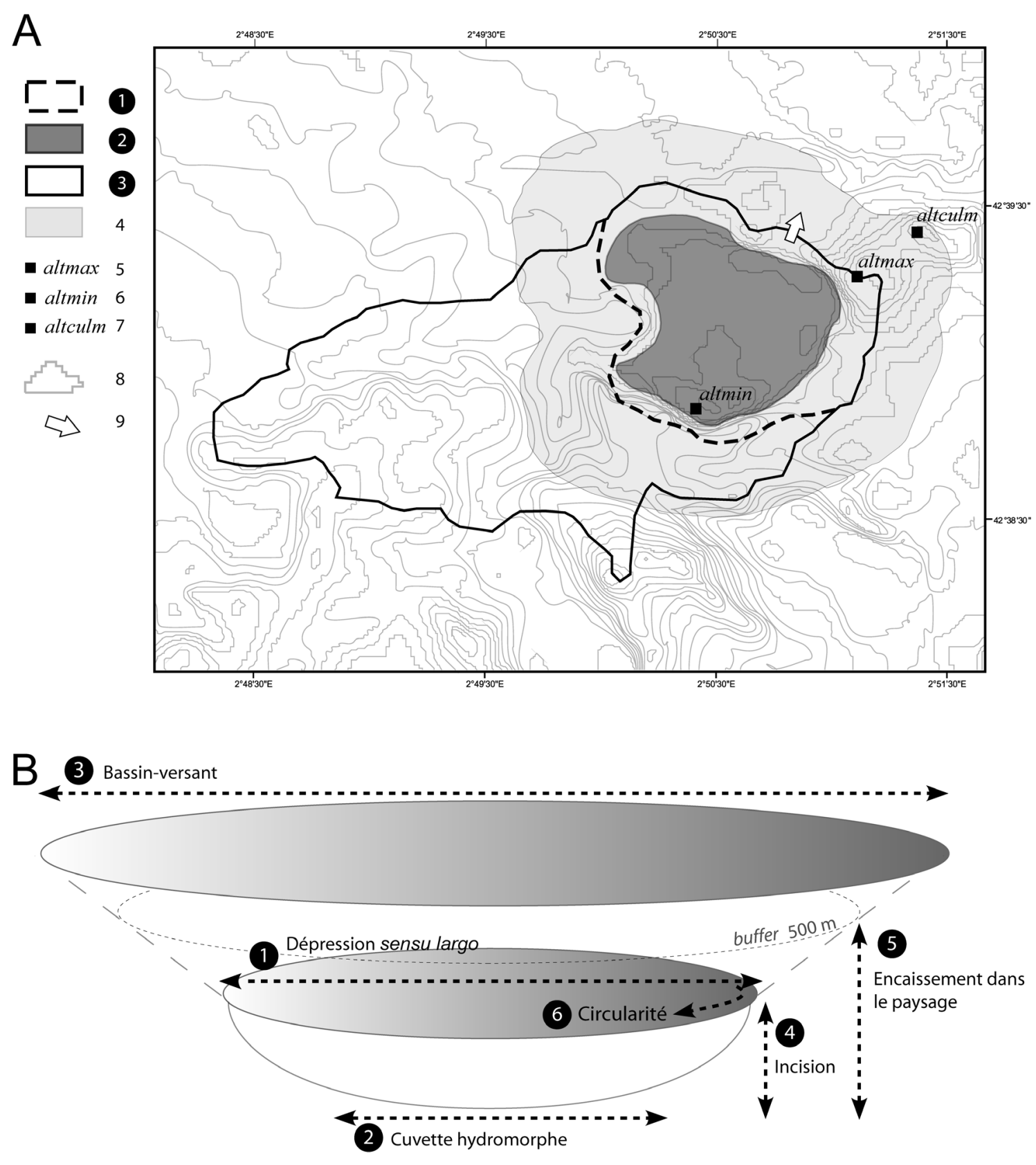

C

\section{Indices morphométriques}

(1) Surface de la forme sensu largo (S, en ha)

(2) Indice de surface de la cuvette hydromorphe (If, en \%)

3 Indice de surface du

3 bassin-versant (Ib, en \%)

(4) Indice d'incision

(5) Indice d'encaissement

5 dans le paysage (Ie, en $\mathrm{mm}^{-2}$ )

6 Indice de Gravelius (Kg)

de

\begin{tabular}{|c|c|c|}
\hline $\begin{array}{l}\text { Type } \\
\text { le calcul }\end{array}$ & Normalisation & $\begin{array}{l}\text { Méthode } \\
\text { de calcul }\end{array}$ \\
\hline automatique & non & \\
\hline automatique & oui & If $=\frac{f}{S} 100$ \\
\hline automatique & oui & $\mathrm{Ib}=\frac{\mathrm{b}}{\mathrm{S}} 100$ \\
\hline semi-automatique & oui & $\mathrm{Ii}=\frac{\text { altmax }- \text { altmin }}{\mathrm{S}}$ \\
\hline semi-automatique & oui & $\mathrm{Ie}=\frac{\text { altculm }- \text { altmin }}{\mathrm{S}}$ \\
\hline semi-automatique & non & $\mathrm{Kg}=0,28 \frac{\mathrm{P}}{\sqrt{\mathrm{S}}}$ \\
\hline
\end{tabular}

Objectif de caractérisation

Taille de la forme

Profil de la forme

Etendue du bassin de drainage

Profondeur de la forme

Inscription de la forme dans le paysage

Circularité de la forme 
lement été utilisée. Les différents supports cartographiques utilisés ont été consultés à partir de l'outil web Géoportail (http://www.geoportail.gouv.fr), qui permet le relevé de coordonnées géographiques exactes et une vérification des informations saisies à partir des images satellite.

\section{Inventaire des formes et constitution de la base de données}

Le travail d'inventaire des formes a été réalisé en deux étapes : la première a consisté à réaliser une synthèse des données connues et des inventaires partiels ; la seconde a consisté au repérage de nouvelles formes. La synthèse des données connues a été effectuée à partir de trois inventaires. Les inventaires de J. Bécat (1977) et de J.-M. Carozza et al. (sous presse), bien que très complets, ne sont que partiels. Ils couvrent uniquement la plaine du Roussillon. L'inventaire du Conservatoire des Espaces Naturels du Languedoc Roussillon (CENLR, 2000) couvre quant à lui l'ensemble de la région Languedoc-Roussillon mais présente un moindre niveau d'exhaustivité.

L'identification de nouvelles formes a été réalisée par couplage de 3 critères de reconnaissance : géologique, géomorphologique et toponymique. Sur les cartes géologiques, les formations associées aux étangs (notées k) ont d'abord été inventoriées. Les dépôts quaternaires de forme circulaire d'origine colluviale (notées $\mathrm{Cx}$ et $\mathrm{Cy}$ ) ou alluviale (notées Fx et Fy) ont ensuite été relevées et certaines d'entre elles ont été requalifiées sur la base de leur morphologie en remblaiement de dépressions endoréiques. Les toponymes indicateurs de plans d'eau (« estany », « estagnot» et « estanyol ») ou de zones humides ( « moulières » et « mouillères ») ont été relevés. Ces données ont été confrontées aux données morphologiques afin de confirmer la présence d'une zone en creux identifiée soit sur les cartes topographiques soit sur le MNT à partir de l'interpolation des isohypses. Toutes ces informations ont été relevées et confrontées entre elles afin de confirmer la nature des formes et d'éliminer les doublons. Les formes ainsi inventoriées ont été localisées sous SIG (ArcGis 9.3).

Les formes inventoriées ont ensuite fait l'objet d'une caractérisation de leur morphologie par une série d'indices morphométriques (fig. 4). Deux types de paramètres ont été pris en considération : les paramètres de la forme sensu largo et les paramètres d'environnement de la forme.

\section{Délimitation et caractérisation de la forme}

Les limites de la cuvette hydromorphe et celles de la forme sensu largo (fig. 4) ont d'abord été tracées de manière semi-automatique par l'application d'un seuillage sur la carte des pentes générée à partir du MNT dans le SIG en utilisant un algorithme de type Zevenbergen et Thorne. Le seuil de transition entre versant et cuvette hydromorphe a été fixé empiriquement à partir de dépressions connues et bien marquées dans le paysage (Canohès, Villeneuve-de-laRaho, Sainte-Croix à Sigean et Montady ; fig. 2, fig. 9 et fig. 10). Le seuil obtenu est variable selon les formes. Il se situe entre $0,5^{\circ}$ et $3^{\circ}$ et dépend de la dynamique de remblaiement de la forme, autrement dit de l'importance respective des processus alluviaux (cônes) et colluviaux (versant). La limite de la forme a ensuite été digitalisée manuellement. Cette méthode a été préférée à une vectorisation automatique car elle permet à l'opérateur une délimitation plus précise.

L'aire et le périmètre de la cuvette hydromorphe (respectivement $\mathrm{f}$ en $\mathrm{m}^{2}$ et $\mathrm{p}$ en $\mathrm{m}$ ) et de la forme sensu largo (respectivement $\mathrm{S}$ en $\mathrm{m}^{2}$ et $\mathrm{P}$ en $\mathrm{m}^{2}$ ) de chaque dépression ainsi délimitée, ont ensuite été calculés automatiquement à l'aide de l'outil SIG (fig. 4). Pour une meilleure caractérisation du profil de la dépression, l'aire de la cuvette hydromorphe (f) a été normalisée (If, en \%) par la surface totale de la forme (S). Pour chaque dépression, les altitudes minimales (altmin, en $\mathrm{m}$ ) et maximales (altmax, en $\mathrm{m}$ ) ont été extraites automatiquement par superposition entre la forme délimitée et le MNT original. Le calcul semi-automatique de la différence entre les deux extrêmes altitudinaux (altmin et altmax), ramenée à la surface de la forme $(\mathrm{S})$, a permis la définition d'un indice d'incision (Ii, en $\mathrm{mm}^{-2}$; fig. 4). Ce dernier caractérise la profondeur relative de la dépression. Enfin, l'indice de compacité de Gravelius (Kg, Roche, 1963) a été calculé (fig. 4) de manière semi-automatique à partir du périmètre $(\mathrm{P})$ et de l'aire (S) de la forme sensu largo. Cet indice correspond au rapport du périmètre de la dépression au périmètre du cercle ayant la même surface soit $\mathrm{Kg}=0,28 \mathrm{P} / \mathrm{A}^{0,5}$. Cet indice donne une indication de la circularité de la forme. L'étude semi-automatique de l'orientation préférentielle des dépressions a été tentée mais se heurte au cas des formes complexes multilobées. Ce critère n'a donc pas été retenu dans l'analyse finale malgré son intérêt.

\section{Délimitation et caractérisation de l'environnement de la forme}

Les dépressions fermées collectent les écoulements de bassins versants plus ou moins vastes. Pour les formes les plus simples, les aires de la forme sensu largo et celle du bassin-versant sont très proches. Dans le cas de formes complexes et évoluées, ce ratio augmente. Les bassins versants drainés vers les dépressions ont été délimités manuellement à partir des courbes isohypses calculées automatiquement d'après le MNT (fig. 4). Cette procédure est nécessaire car il n'est pas possible d'appliquer l'algorithme automatique de calcul du bassin versant qui n'a pas d'exutoire unique. Leur surface $\left(b\right.$, en $\left.\mathrm{m}^{2}\right)$ a ensuite été calculée automatiquement et normalisée ( $\mathrm{Ib}$, en \%) par la surface de la forme (S ; fig. 4). Afin de caractériser la position de la forme dans son environnement, son inscription topographique dans le paysage a été recherchée. Pour cela, un buffer de $500 \mathrm{~m}$ autour de la forme sensu largo a systématiquement été tracé. L'altitude du point culminant de cette surface (altculm, en m) a été recherchée par superposition. Le calcul de la différence entre le point culminant du buffer (altculm) et le point le plus bas de la forme (altmin), normalisé par la surface de la forme $(\mathrm{S})$, a permis de définir un indice d'encaissement (Ie, en $\mathrm{mm}^{-2}$; fig. 4). Ce dernier indice caractérise l'inscription de la forme dans le paysage et oppose les formes dévelop- 
pées sur les plateaux et les formes de fond de talweg. Enfin, la lithologie sur laquelle la forme se développe a été renseignée. Cependant, cet indicateur qualitatif unique n'a pas été intégré dans la typologie finale.

\section{Traitement des données}

L'ensemble des informations ainsi obtenues a fait l'objet de deux types d'analyses. D'une part, l'analyse de la variabilité des indices morphométriques calculés a permis de mettre en évidence la dispersion de chacun des descripteurs (analyse univariée). D'autre part, les variables ont été centrées et réduites afin de réaliser une Analyse en Composante Principale (ACP). L'objectif de l'ACP est de regrouper les variables redondantes et de discriminer les facteurs les plus influents. Une typologie a ensuite été réalisée par regroupement des individus sur les deux premiers axes factoriels F1 et F2. L'ACP a été réalisée à l'aide du module statistique du logiciel Philcarto (Wanniez, 2008).

\section{Résultats}

\section{Inventaire des formes}

Un corpus de 175 dépressions fermées a été constitué pour l'ensemble du territoire Languedoc central/Roussillon, parmi lequel 61 formes (soit

Fig. 5 - Inventaire et délimitation des dépressions fermées. A : Inventaire des formes. 175 dépressions ont été inventoriées, parmi lesquelles 61 n'avaient pas été relevées dans les inventaires précédents. L'histogramme montre l'évolution du travail d'inventaire sur les basses plaines du Languedoc central et du Roussillon entre 1977 et l'actuel, avec les travaux de J. Bécat, 1977 (Roussillon), CENLR, 2000 (région LanguedocRoussillon) et J.-M. Carozza et al., 2010, sous presse (Roussillon). B : Délimitation des formes. Sur les 175 dépressions inventoriées, 83 ont pu être délimitées. Le diagramme circulaire montre plus en détail la proportion des formes inventoriées qui ont pu être délimitées. 1 : dépressions délimitées qui n'avaient pas été relevées dans les inventaires précédents ; 2 : dépressions délimitées reconnues dans les inventaires précédents ; 3 : dépressions non délimitées.

Fig. 5 - Inventory and delimitation of endorheic depressions. A: Depressions inventory. 175 depressions have been identified, 61 of which did not appear in previous inventories. The development of the inventory work in lowlands of central Languedoc and Roussillon between 1977 and today is shown in the histogram with the studies of J. Bécat, 1977 (Roussillon), CENLR, 2000 (Languedoc-Roussillon region), and J.-M. Carozza et al., 2010, in press (Roussillon). B: Depressions delimitation. Of the remaining 175, 83 depressions were characterised by adequate size parameters necessary for further analysis. Full details on depressions that were delimited are shown in the pie chart. 1: delimited depressions which did not appear in previous inventories; 2: delimited depressions which appear in previous inventories; 3: depressions which are not delimited.
$35 \%$ de l'effectif total) n'avaient jusqu'alors jamais été identifiées dans la littérature. La carte de répartition des dépressions relevées (fig. 5) montre une concentration préférentielle des formes sur l'interfluve Têt/Tech, le secteur de Sigean et les vallées de l'Aude et de l'Orbieu. Sur l'effectif total, 83 dépressions (47\%) sont suffisamment grandes et/ou marquées dans le paysage pour permettre un travail de délimitation suffisamment précis à partir des sources documentaires utilisées (fig. 5). La résolution spatiale du MNT utilisé est le principal facteur limitant qui a conduit à écarter les dépressions les plus petites. Les autres formes n'ont pas été prises en compte dans la suite de cette analyse morphométrique.
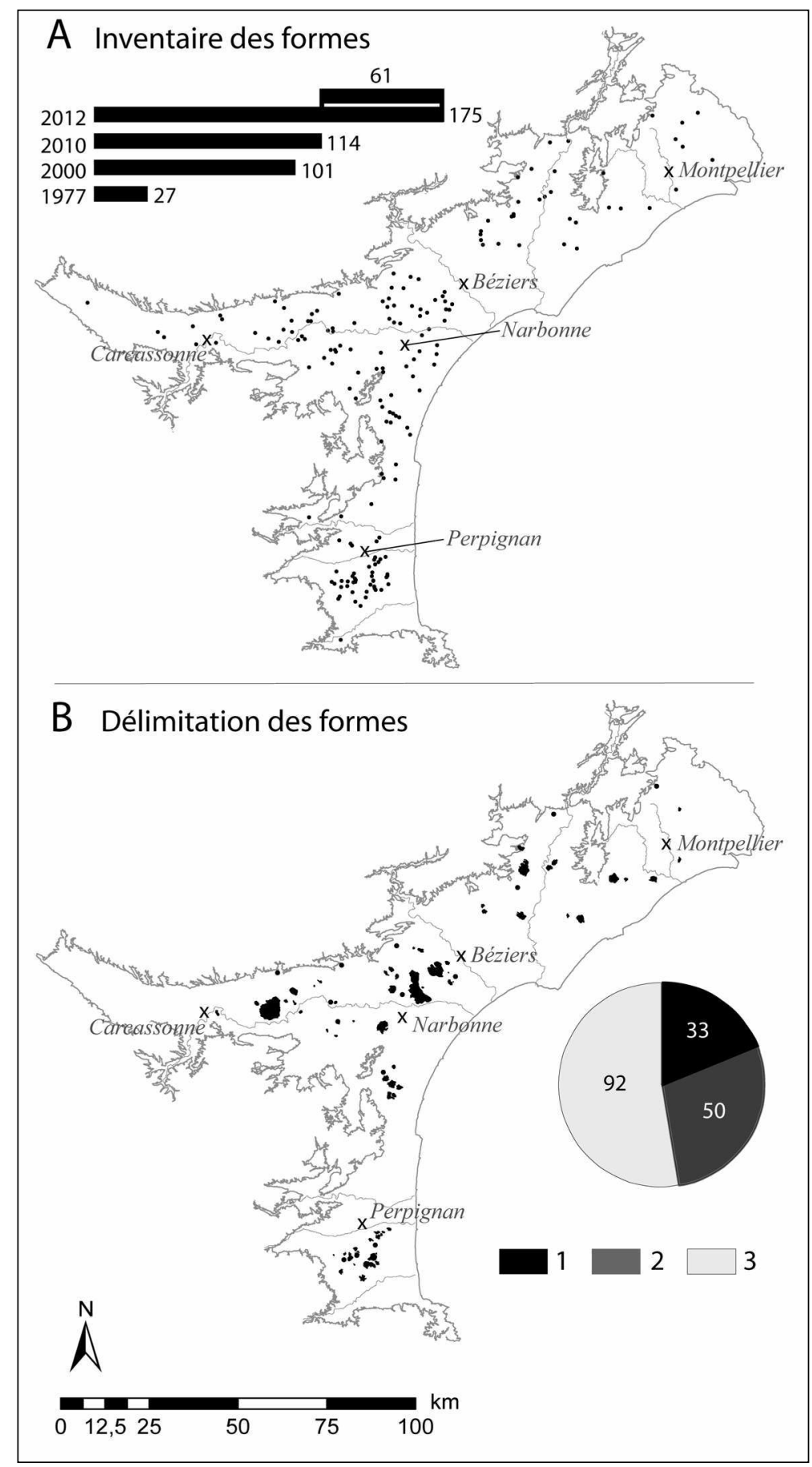


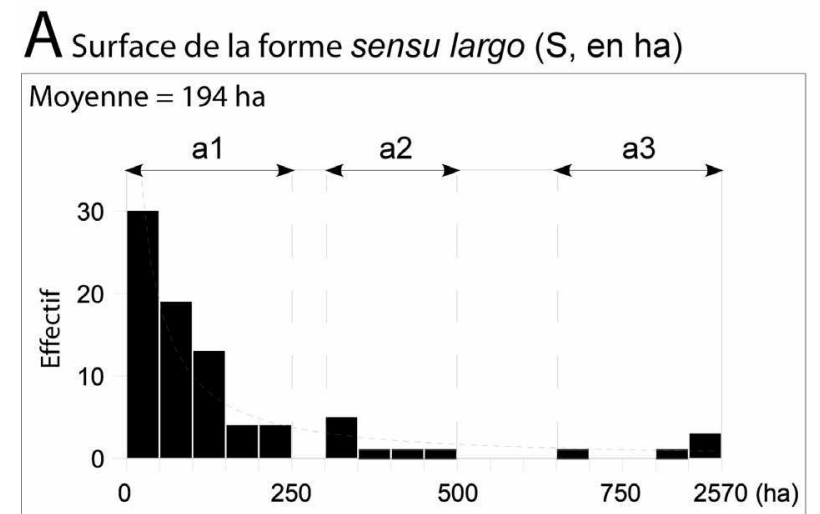

B Indice de surface de la cuvette hydromorphe (If, en \%) Moyenne $=25,95 \%$

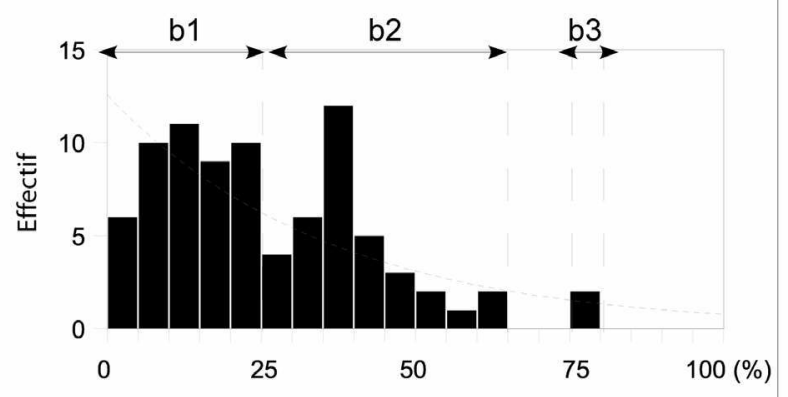

C Indice de surface du bassin versant (Ib, en \%) Moyenne $=353 \%$

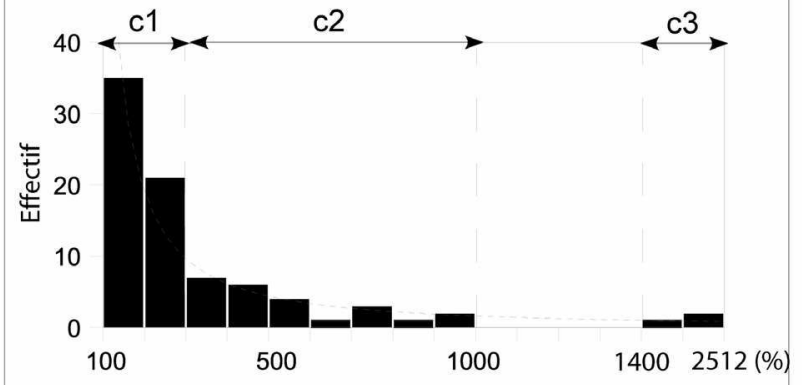

E Indice d'encaissement dans le paysage (le, en $\mathrm{mm}^{-2}$ )

D Indice d'incision (li, en $\mathrm{mm}^{-2}$ )

Moyenne $=0,46 \mathrm{~m} \cdot \mathrm{m}^{2}$

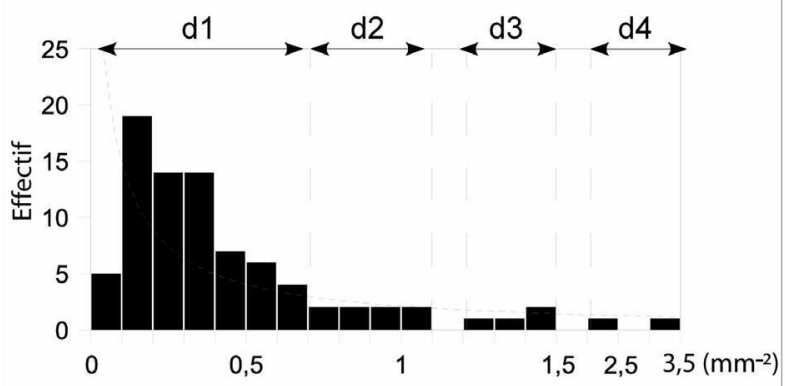

$\mathrm{F}_{\text {Indice de Gravelius (Kg) }}$

Moyenne $=0,80 \mathrm{~m} \cdot \mathrm{m}^{2}$

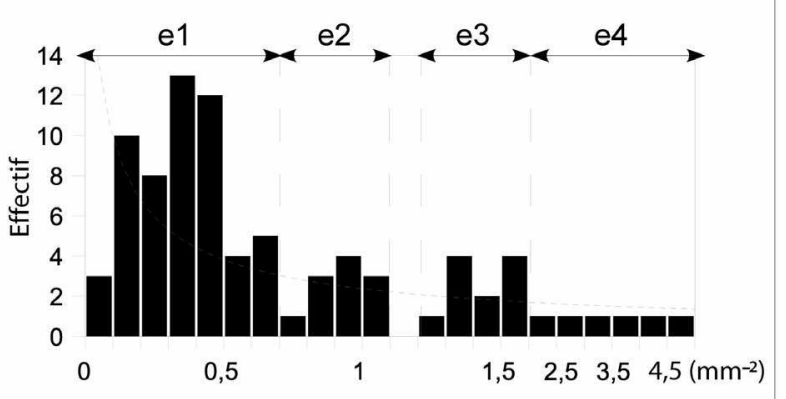

Moyenne $=1,16$

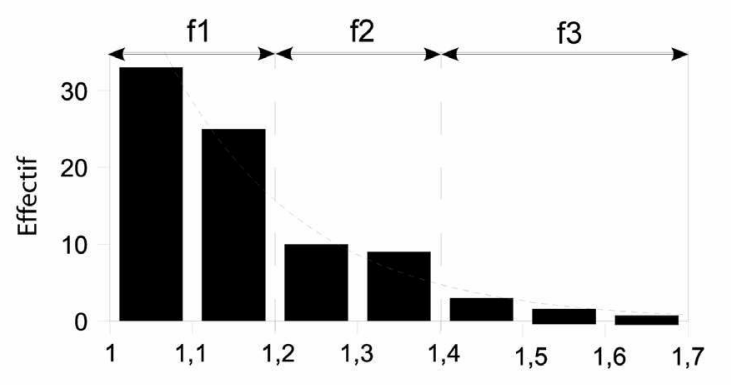

Fig. 6 - Analyse univariée des formes selon six indicateurs morphométriques. Pour chaque indicateur, un histogramme présente les valeurs de l'indice en abscisse et le nombre de dépressions en ordonnée. La valeur moyenne est indiquée sur chaque cadre et des groupes de dépressions, définis à partir de seuils dans la distribution, sont délimités par des flèches horizontales. A : Surface de la dépression sensu largo (S, en ha). B : Indice de surface de la cuvette hydromorphe (If, en \%). C : Indice de surface du bassin-versant (Ib, en \%). D : Indice d'incision (li, en $\left.\mathrm{mm}^{-2}\right)$. E : Indice d'encaissement dans le paysage (le, en $\left.\mathrm{mm}^{-2}\right)$. F : Indice de Gravelius (Kg, tendance à la circularité si l'indice est proche de 1 , tendance inverse si l'indice est proche de 2).

Fig. 6 - Univariate analysis of depressions with six morphometric parameters. For each parameter, the graph indicates the value of the parameter in abscissa and the number of depressions in ordinate. The mean value appears at the top of the graph and groups of depressions, which are defined by thresholds in the distribution, are shown with horizontal arrows. A: Area of the depression sensu largo (S, in ha). $B$ : Area of the hollow wetland index (If, in \%). C: Area of the catchment index (Ib, in \%). D: Incision index (li, in mm-2). E: Index of shape incision in the landscape (le, in $\mathrm{mm}^{-2}$ ). F: Circularity index ( $\mathrm{Kg}$, a value tending toward 1 means the shape is close to circularity; a value tending toward 2 means the shape is not close to circularity).

\section{Analyse morphométrique}

Traitement statistique univarié

Pour les 83 dépressions qui ont pu être délimitées et caractérisées, six indices descriptifs morphométriques ont été calculés. Les résultats de cette analyse sont présentés sous forme d'histogrammes (fig. 6) et une répartition des individus a pu être faite à partir de l'étude des modes statistiques.

L'histogramme de répartition des dépressions selon leur taille (S, en ha ; fig. 6A) montre une prédominance des petites dépressions ( $11: \mathrm{S}<250$ ha) et notamment des formes 
les plus réduites, qui mesurent moins de 50 ha (e.g., l'étang de la Cardaïro à Cruscades et l'estany del Comte à Ponteilla ; fig. 9). Deux groupes apparaissent ensuite : un groupe de formes de tailles comprises entre 300 et 500 ha (a2) et un groupe de formes particulièrement étendues, de tailles comprises entre 650 et plus de 2500 ha (a3; e.g., les étangs de Marseillette, Capestang et Montady ; fig. 2, fig. 9 et fig. 10).

L'histogramme de distribution des dépressions selon l'étendue de leur cuvette hydromorphe (lf, en \% de la forme ; fig. 6B) présente une répartition bimodale avec un groupe de formes pour lesquelles la cuvette hydromorphe représente une faible proportion de la forme (b1 : If $<25 \%$ ) et un groupe de formes pour lesquelles la cuvette hydromorphe est plus étendue (b2 : lf $=25-65 \%$ ). Deux formes atypiques présentent des cuvettes hydromorphes très étendues (b3 : If = 75-80 \%). Il s'agit des étangs de Capestang et de Marseillette (fig. 9).

L'histogramme de distribution des dépressions selon l'étendue de leur bassin-versant (lb, en \% de la forme ; fig. 6C) montre une surreprésentation des formes à petit bassin-versant très concentré autour de la forme, qui représentent moins de 3 fois la surface de la forme sensu largo $(\mathrm{c} 1: \mathrm{lb}=$ 100-300 \%). La distribution est cependant étendue, avec des bassins versants couvrant jusqu'à 10 fois la forme (c2 : lb =
$300-1000 \%$ ) et quelques individus atypiques, qui se distinguent avec des bassins versants représentant plus de 14 fois la taille de la forme (c3 : lb = 1 400-2 $512 \%$; e.g., l'Étang du Bernat à Colombiers et les Prats à Thuir ; fig. 9).

Les histogrammes de répartition des dépressions selon les indices d'incision ( $\mathrm{Ii}$, en $\mathrm{mm}^{-2}$; fig. 6D) et d'encaissement dans le paysage (Ie, en $\mathrm{mm}^{-2}$; fig. $6 \mathrm{E}$ ) ont un aspect relativement proche, avec une distribution plus étalée pour l'indice d'encaissement. Dans l'ensemble, les formes sont peu marquées dans le paysage, avec un indice d'incision et/ou d'encaissement dominant inférieur à $0,7 \mathrm{~mm}^{-2}(\mathrm{~d} 1$ et e1). Cependant, quelques regroupements de formes s'inscrivent plus nettement dans le paysage avec un indice d'incision pouvant aller jusqu'à $3,5 \mathrm{~mm}^{-2}\left(\mathrm{~d} 2: \mathrm{Ii}=0,7-1,1 \mathrm{~mm}^{-2} ; \mathrm{d} 3\right.$ : $\left.\mathrm{Ii}=1,2-1,5 \mathrm{~mm}^{-2} ; \mathrm{d} 4: \mathrm{Ii}=2-3,5 \mathrm{~mm}^{-2}\right)$ et/ou avec un indice d'encaissement maximal de presque $5 \mathrm{~mm}^{-2}(\mathrm{e} 2: \mathrm{Ie}=$ 0,7-1,1 $\mathrm{mm}^{-2}$; e3 : Ie =1,2-2 $\mathrm{mm}^{-2}$; e4 $\left.: \mathrm{Ie}=2-5 \mathrm{~mm}^{-2}\right)$. Poussan-le-Haut à Béziers et Le Clot à Nizas (fig. 9 et fig. 10) illustrent bien le cas des formes les plus incisées (resp. Ii $=3,33 \mathrm{~mm}^{-2}$ et $\mathrm{Ii}=2,14 \mathrm{~mm}^{-2}$ ) et les plus encaissées dans le paysage (resp. Ie $=2,14 \mathrm{~mm}^{-2}$ et Ie $=3,36 \mathrm{~mm}^{-2}$ ).

L'analyse des formes en fonction de l'indice de circularité de Gravelius ( $\mathrm{Kg}$; fig. 6F) révèle une surreprésentation

$\mathrm{F} 1$ et $\mathrm{F} 2=65,79 \%$ de la variance

F2 [17,62 \%]

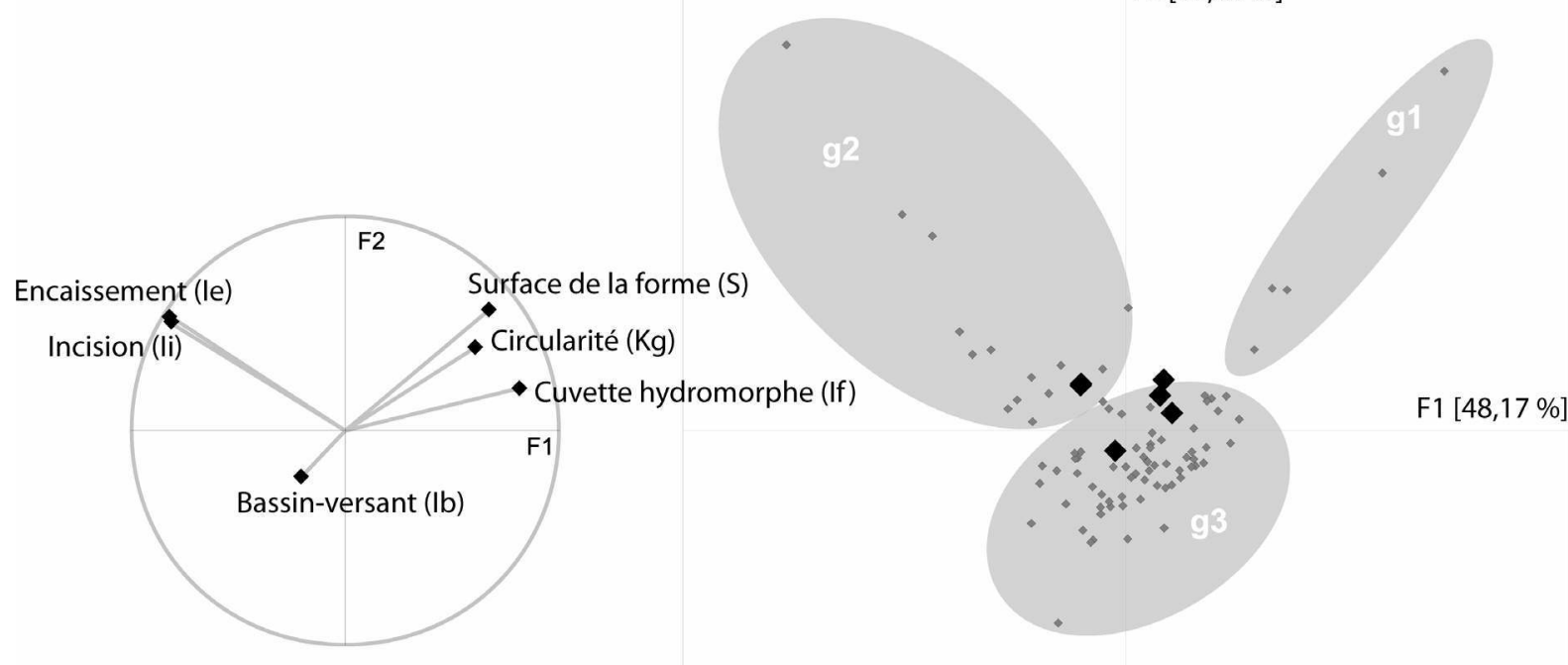

Fig. 7 - Cercle des corrélations et projection des points sur les axes factorielles (ACP). Les axes factoriels F1 et F2 expliquent 65,79 \% de la variance observée. Le cercle des corrélations (à gauche) montre que deux groupes de variables caractérisent l'axe factoriel F1 $(48,17 \%$ de la variance) : groupe 1 : indices d'incision (li) et d'encaissement (le); groupe 2 : indice de surface de la cuvette hydromorphe (If), surface de la forme sensu largo (S) et indice de Gravelius (Kg). Sur l'axe factoriel F2 (17,62\% de la variance), seul l'indice de surface du bassin-versant (Ib) représente un poids significatif. II est anti-corrélé avec les autres variables. La projection des individus sur les axes $\mathrm{F} 1$ et F2 (à droite) montre un regroupement en trois ensembles : g1 : 5 individus ; g2 : 14 individus et g3 $: 64$ individus. Les variables sont figurées sur le nuage de point par des carrés.

Fig. 7 - Circle of correlations and projection of points on the factorial axes (PCA). The factorial axes F1 and F2 explain $65,79 \%$ of the observed variance. The circle of correlation (left) shows that the factorial axe $F 1(48,17 \%$ of the variance) is characterised by two groups of parameters: on one side parameters related to the vertical dimension of the depressions (Incision index, li, and Index of shape incision in the landscape, le) and on the other, parameters related to the horizontal dimension of the depressions (area of the hollow wetland index, If; area of the depression sensu largo, $\mathrm{S}$, and circularity index, $\mathrm{Kg}$ ). Only the area of the catchment index (Ib) characterises the factorial axe F2 $(17,62 \%$ of the variance). Ib anti correlates with other parameters. The projection of points on the factorial axes F1 and F2 (right) shows three groups: g1: 5 units; g2: 14 units; G3: 64 units. The parameters are represented on the cloud point by squares. 


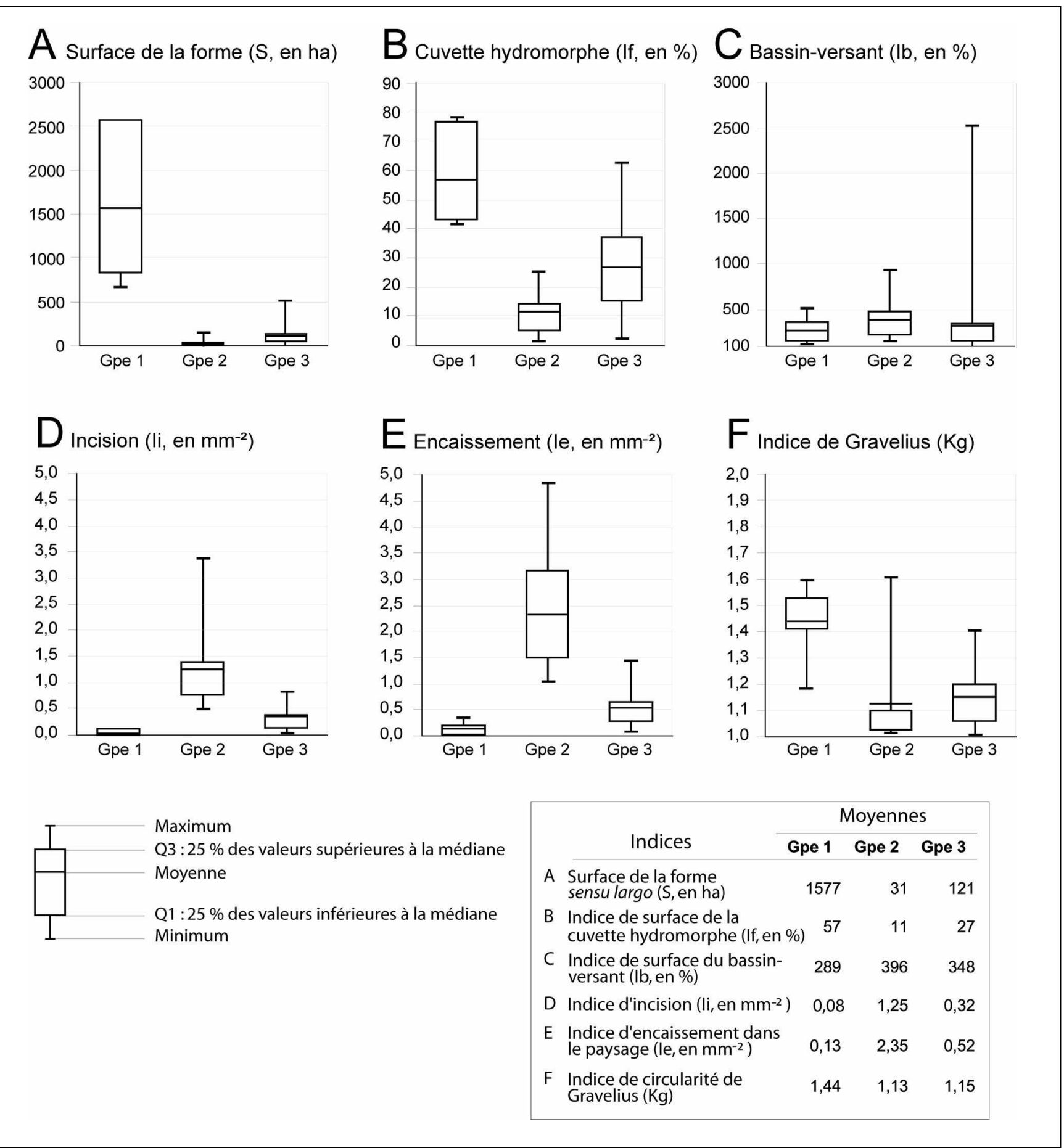

Fig. 8 - Caractérisation des groupes de forme en fonction des indicateurs morphométriques. Pour chaque indicateur, un diagramme en boite présente la distribution des dépressions au sein des trois groupes de formes définis par l'ACP. La ligne verticale définit l'étendue de la population statistique ; la boite indique le 1er quartile (Q1 : $25 \%$ des valeurs sont inférieures à la médiane) et le 3 ème quartile (25\% des valeurs sont supérieures à la médiane); la ligne horizontale situe la valeur moyenne. Les valeurs moyennes des indicateurs sont détaillées pour chaque groupe dans le tableau au bas de la figure. A : Surface de la dépression sensu largo (S, en ha). B : Indice de surface de la cuvette hydromorphe (If, en \%). C : Indice de surface du bassin-versant (Ib, en \%). D : Indice d'incision (li, en $\mathrm{mm}^{-2}$ ). E : Indice d'encaissement dans le paysage (le, en $\left.\mathrm{mm}^{2}\right)$. $\mathrm{F}$ : Indice de Gravelius ( $\mathrm{Kg}$, tendance à la circularité si l'indice est proche de 1 , tendance inverse si l'indice est proche de 2).

Fig. 8 - Characterisation of groups of forms with morphometric parameters. For each indicator a box plot shows the distribution of depressions inside the three groups that were defined by performing PCA. A vertical line defines the extent between the maximum and minimum values; a rectangle shows the first quartile (Q1: $25 \%$ of the values is bellow the median) and the third quartile (Q3: $25 \%$ of the values is above the median) and a horizontal line shows the mean value. For each group, mean values are indicated in the table at the bottom of the figure. A: Area of the depression sensu largo (S, in ha). B: Area of the hollow wetland index (If, in \%). C: Area of the catchment index (Ib, in \%). D: Incision index (li, in $\mathrm{mm}^{2}$ ). E: Index of shape incision in the landscape (le, in $\mathrm{mm}^{2}{ }^{2}$ ). F: Circularity index (Kg, a value tending toward 1 means the shape is close to circularity; a value tending toward 2 means the shape is not close to circularity). 
des dépressions qui tendent vers la circularité. La majeure partie des formes présente en effet un indice inférieur à 1,4 (f1 : Kg < 1,2; f2 : Kg = 1,2-1,4) avec une nette surreprésentation des formes les plus circulaires (f1). Quelques formes présentent cependant un indice relativement fort (f3: $\mathrm{Kg}=$ 1,4-1,7). C'est notamment le cas des dépressions emblématiques de Capestang $(\mathrm{Kg}=1,61)$, Montady $(\mathrm{Kg}=1,41)$ et Bages $(\mathrm{Kg}=1,40)$, ainsi que de l'Étang de Fontes $(\mathrm{Kg}=$ 1,53 ; fig. 2, fig. 9 et fig. 10). L'interprétation de cet indice doit prendre en compte la résolution du MNT à partir duquel la vectorisation a été effectuée. En effet, la géométrie des dépressions les plus petites est influencée par l'effet de pixellisation. Les contours des plus petites dépressions en sont simplifiées et tendent généralement vers la circularité. Les contours des dépressions les plus grandes, telles que celles de Montady ou de Bages, sont susceptibles d'être mieux représentés dans leur complexité. Cependant, leur forme globale tend vers la circularité. Du fait de cette limite, seules les formes qui présentent un indice supérieur à 1,5 présentent ici un réel allongement, indépendamment de la sinuosité du contour. En dessous de ce seuil, les nuances de valeurs entre les formes sont essentiellement dues à la sinuosité des contours, particulièrement marquée pour les formes les plus grandes. C'est le cas des étangs de Capestang et de Fontes (fig. 9).

Les formes identifiées dans l'inventaire sont principalement développées sur des terrains de molasse sableuse plus ou moins carbonatée du Miocène ou du Pliocène. Cependant dans le détail, six substrats différents se sont révélés favorables au développement de dépressions fermées : Quaternaire, Pliocène, Miocène, Oligocène, Eocène et Trias (fig. 3).

\section{Traitement statistique multivarié}

Une analyse en composantes principales (ACP) a été réalisée sur la base de données afin de discriminer des groupes de formes aux caractères morphométriques similaires.

Les deux premiers axes factoriels F1 et F2 expliquent $65,79 \%$ de la variance observée (fig. 7). Les scores factoriels montrent que deux groupes de variables caractérisent l'axe factoriel F1 (48,17\% de la variance). Le premier groupe renferme les indices associés à la dimension verticale des formes (indices d'incision et d'encaissement). Le second groupe correspond aux indicateurs de dimension et de forme en plan (indice de surface de la cuvette hydromorphe, surface de la forme sensu largo et indice de Gravelius). Sur l'axe factoriel F2 (17,62 \% de la variance), seul l'indice de surface du bassin-versant présente un poids significatif. Il est anti-corrélé avec les autres variables. La projection des individus sur les axes $\mathrm{F} 1$ et $\mathrm{F} 2$ montre un regroupement en trois ensembles (fig. 7 et fig. 8) :

- Le premier groupe $(\mathrm{g} 1)$ correspond à cinq individus de grande taille avec un indice de Gravelius fort (donc de forme allongée ou complexe) et des cuvettes hydromorphes très étendues. La taille moyenne des formes du groupe g1 (Smoy = 1577 ha) est en effet nettement supérieure à celles des deux autres groupes. De plus, $50 \%$ des formes du groupe g1 présentent une surface supérieure à 700 ha alors que toutes les formes des autres groupes mesurent moins de 500 ha.
Les cuvettes hydromorphes des dépressions du groupe g1 couvrent toutes plus de $40 \%$ de la forme et peuvent couvrir jusqu'à $80 \%$ de la forme, ce qui est nettement supérieur aux moyennes des groupes g2 et $\mathrm{g} 3$ (respectivement fmoy $=$ $11 \%$ et lfmoy $=27 \%$ ). Les formes du groupe g1 présentent enfin un indice de Gravelius moyen $(\mathrm{Kgmoy}=1,44)$ nettement supérieur à celui des groupes g1 et g2 (respectivement Kgmoy = 1,13 et Kgmoy = 1,15).

- Le deuxième groupe $(\mathrm{g} 2)$ correspond aux formes fortement incisées (Iimoy $=1,25 \mathrm{~mm}^{-2}$ ) et encaissées dans le paysage $\left(\right.$ Iemoy $\left.=2,35 \mathrm{~mm}^{-2}\right)$. Il est anti-corrélé avec le groupe g1, ce qui signifie qu'il rassemble des formes de taille réduite (Smoy $=31 \mathrm{ha}$ ), à cuvette hydromorphe peu étendue (fmoy $=11 \%$ ) et qui présentent un indice de Gravelius fort. L'indice de Gravelius moyen pour le groupe g2 est en réalité plus faible que pour les autres groupes (moyenne $: \mathrm{Kg}=1,13$ ) mais ce groupe est plus hétérogène que les autres avec un indice maximum égal à celui du groupe g1 $(\operatorname{Kgmax}=1,6)$.

- Le troisième groupe (g3) comprend la majorité des dépressions inventoriées, soit 64 formes. Il rassemble les formes anti-corrélées avec les deux autres groupes et caractérisées par un bassin-versant étendu. Ces formes ne sont pas très marquées dans le paysage (Iimoy $=0,32 \mathrm{~mm}^{-2}$; Iemoy $=$ $0,52 \mathrm{~mm}^{2}$ ) et présentent des tailles plutôt réduites avec une moyenne de 121 ha et un maximum de 500 ha. Cependant, leurs cuvettes hydromorphes sont relativement étendues (lfmoy $=27 \%$; lfmax $>60 \%$; lfQ3 = $35 \%$ ). La plupart des bassins versants du groupe g3 est plutôt réduite (lbmoy = $348 \% ; \mathrm{lbQ} 3=350 \%$ ) mais ce groupe présente des bassins versants hétérogènes qui peuvent représenter jusqu'à 25 fois la surface de la forme (lbmax $=2500 \%$ ).

La spatialisation des groupes de formes (fig. 9) montre que les dépressions du groupe g1 se concentrent essentiellement dans le couloir de la vallée de l'Aude tandis que la répartition des deux autres groupes est plus hétérogène.

\section{Interprétation et discussion}

La dispersion des indicateurs ainsi que l'existence de trois groupes de formes identifiés par l'ACP mettent en évidence la diversité morphométrique des dépressions fermées du Languedoc central et du Roussillon. Celle-ci aurait probablement été encore accrue si l'ensemble du corpus des 175 formes avait été pris en considération dans ce travail et avait inclut les formes de petite taille. Dans ces conditions, attribuer l'ensemble des formes observées sur ce territoire à un processus morphogène unique paraît difficile.

La déflation éolienne est susceptible d'être à l'origine des formes les plus étalées, les moins encaissées et les plus complexes qui se concentrent dans le couloir d'accélération de la Tramontane (vallée de l'Aude), comme c'est le cas de l'Étang de Capestang (fig. 9). Ces dépressions constituent l'essentiel du groupe g1. Il est également possible que la déflation nivo-éolienne soit à l'origine de la coalescence de formes initialement distinctes et qui se retrouvent partiellement dans le groupe g3. Ainsi, l'ensemble multilobé Retenue d'eau / Estagnol à Villeneuve-de-la-Raho (fig. 9) pour- 


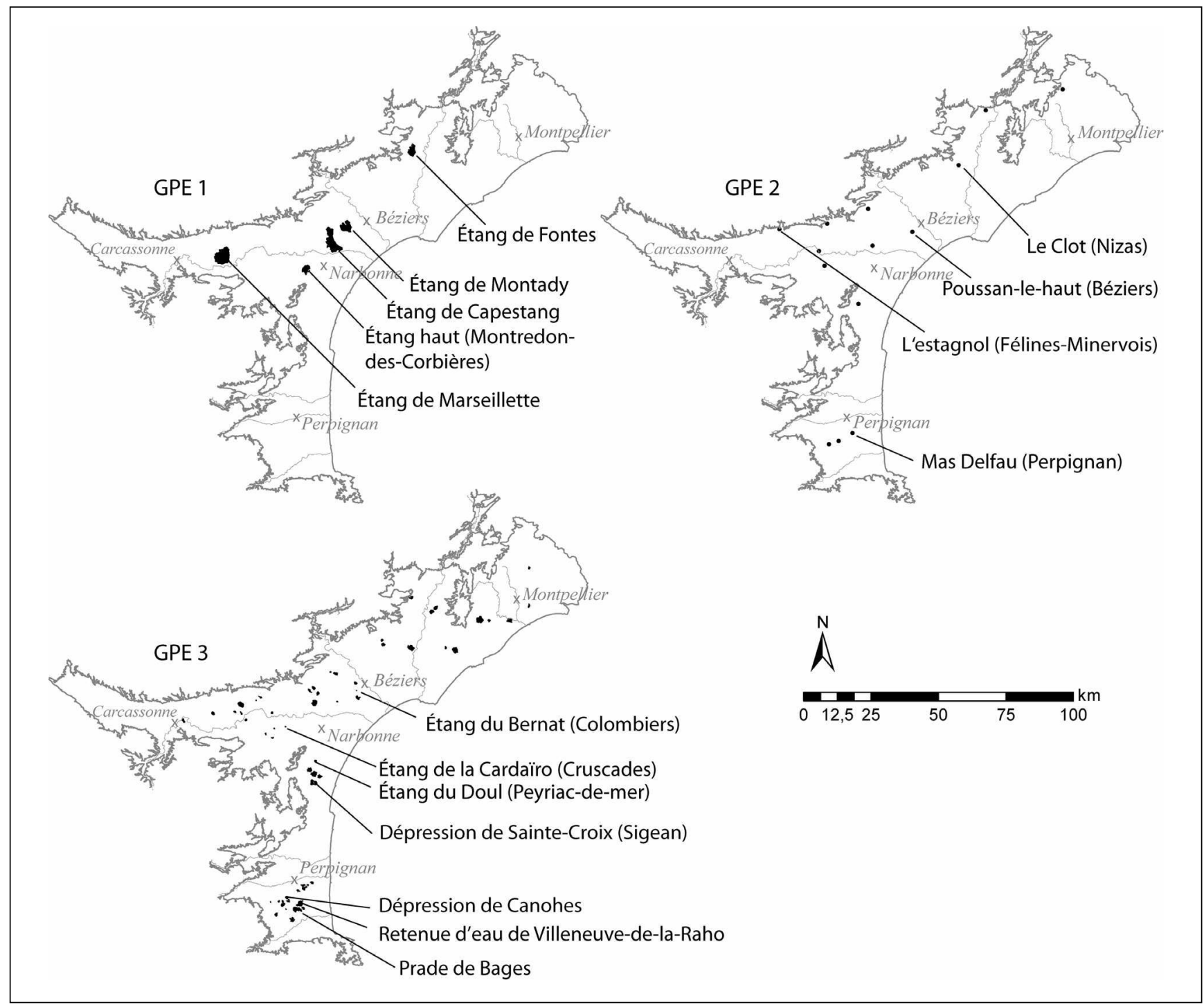

Fig. 9 - Distribution spatiale de la typologie morphométrique. Pour chacun des trois groupes de formes définis par l'ACP, une carte présente la distribution spatiale des dépressions. GPE 1 : dépressions du groupe g1 - les cinq formes sont délimitées et nommées. GPE 2 : dépressions du groupe g2 - les 14 formes sont localisées par un point car l'échelle de la figure ne permet pas une bonne visibilité de leurs contours ; dans un souci de clarté, seules les dépressions données en exemple dans le texte sont nommées. GPE 3 : dépressions du groupe g3 - les 64 formes sont délimitées ; dans un souci de clarté, seules les dépressions données en exemple dans le texte sont nommées.

Fig. 9 - Spatial distribution of the morphometric typology results. For each of the three groups that were defined by performing PCA, a map shows the spatial distribution of the depressions. GPE 1: The five depressions of group g1. The forms are delimited and the names are given. GPE 2: The 14 depressions of group g2. As the map scale does not reveal small shapes, the depressions are indicated by a dot. For reasons of clarity, only the names of depressions that are mentioned in the text appear on the map. GPE 3: The 64 depressions of group g3. All the forms are delimited. For reasons of clarity, only the names of depressions that are mentioned in the text appear on the map.

rait résulter de l'agrégation de formes plus petites par ce type de processus et/ou par érosion hydrique simple. La déflation peut aussi être à l'origine de formes réduites, façonnées sur les terrasses quaternaires, faiblement encaissées en valeur absolue mais qui présentent un indice d'encaissement fort, à l'image du groupe g2 (e.g., Le Clot à Nizas ; fig. 9 et fig. 10).

Le rôle de la déflation dans la genèse des dépressions circulaires qui présentent généralement des versants abrupts sub-verticaux sans relation avec l'orientation du vent, comme c'est le cas de la dépression de Canohes ou de la dépression de Sainte-Croix à Sigean (fig. 2, fig. 9 et fig. 10), semble plus difficile à accepter. S'il n'est exclu ni une retouche éolienne à ces formes représentatives du groupe g3, ni un rôle de la déflation dans la vidange partielle de ces réservoirs lors du dernier glaciaire, l'absence d'orientation privilégiée et de forme en cupule semble exclure les processus éoliens comme facteurs premiers de formation. En revanche, l'érosion hydro-éolienne semble avoir joué un rôle dans la formation de niveaux de pavages grossiers de dreikanters quartzitiques ou de marbre fréquemment observés (cas de la dépression de Bages ou de Mas Delfau ; fig. 2 et fig. 9). Ces derniers pourraient correspondre soit à des dépôts alluviaux 

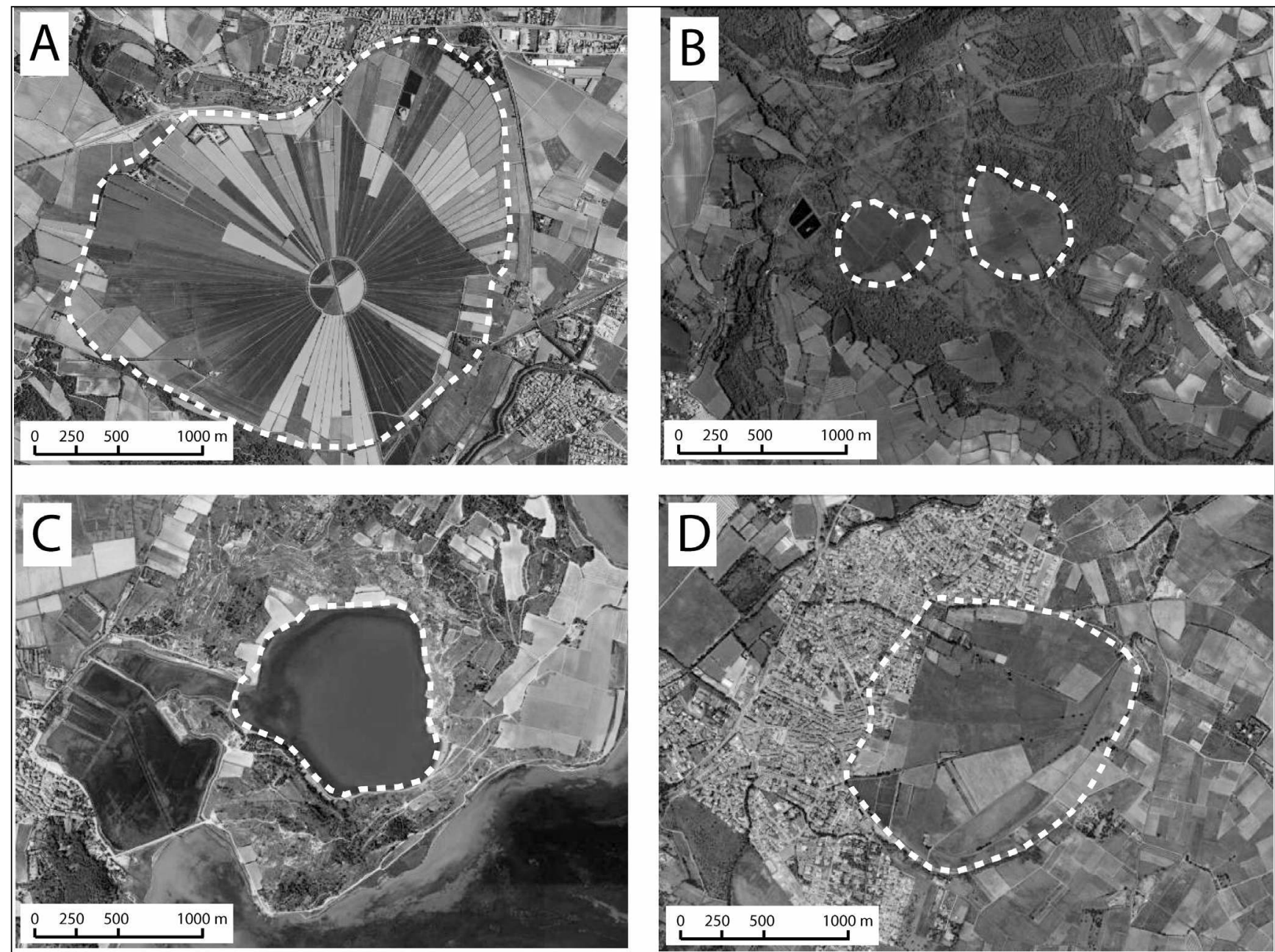

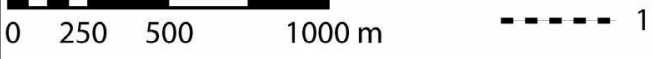

Fig. 10 - Photographies aériennes de quelques dépressions du Languedoc central et du Roussillon (Montady, Caux, Nizas, L'Étang du Doul à Peyriac-de-mer et Canohes, données IGN http://www.geoportail.gouv.fr). 1 : délimitation de la forme sensu largo. A : Dépression de Montady (g1, réseau parcellaire de drainage radioconcentrique médiéval, Hérault). B : L'Estang à Caux (à gauche : non délimité, Hérault) et Le Clot à Nizas (à droite : g2, Hérault). C : L'Etang du Doul à Peyriac-de-mer (g3, origine diapirique attestée, Aude). D : Dépression de Canohes (g3, Pyrénées-Orientales). Ces formes sont localisées sur la fig. 9.

Fig. 10 - Aerial photographs of some enclosed depressions of central Languedoc and Roussillon (Montady, Caux, Nizas, L'Étang du Doul in Peyriac-de-Mer, and Canohes, data from IGN http://www.geoportail.gouv.fr). 1: depression sensu largo. A: Depression of Montady (g1, radioconcentric plot network derived from medieval works, Hérault). B: L'Estang in Caux (left: not possible to analyse with SIG because of insufficient size) and Le Clot in Nizas (right: g2, Hérault). C: L'Etang du Doul in Peyriac-de-Mer (g3, diapir, Aude). D: Depression of Canohes (g3, Pyrénées-Orientales). The locations of these depressions are given in fig. 9.

résiduels remaniés piégés dans la dépression par la déflation, soit à des phases de réactivation des processus de versant en lien avec des épisodes de réchauffement (interglaciaires ou interstadiaires). Ces dernières formes, à l'image de nombreuses autres du groupe $\mathrm{g} 3$, semblent se rapprocher de la géométrie de l'Étang du Doul (fig. 2, fig. 9 et fig. 10) dont l'origine diapirique est attestée de manière certaine (Lespinasse, 1982). S'il faut rester prudent, l'hypothèse d'une telle origine pour certaines des formes des groupes $\mathrm{g} 1$ et g3 pourrait cependant être envisagée. De nombreuses dépressions fermées de ces groupes montrent en effet des niveaux de sel importants qui se traduisent par le développe- ment de végétations halines, voire d'une exploitation du sel lors d'épisodes de sécheresse exceptionnelle (cas de Villeneuve-de-la-Raho ; Martzluff, 1993, ou de Montady ; Abbé, 2006, 2009 ; fig. 2 , fig. 9 et fig. 10). Si ces formations de sel peuvent être d'origine partiellement évaporale, elles pourraient également traduire la présence de niveaux salifères en sub-surface. La coïncidence entre la présence des dépressions circulaires les plus grandes du groupe g3 et des secteurs de faille néogènes majeures, comme autour de la faille de Thuir en Roussillon ou de la faille de Portel en Languedoc, supporte cette interprétation (Collina-Girard, 1989). La diversité des formes associée au groupe g3 peut être inter- 
prétée comme la signature de l'évolution des formes postérieurement à leur façonnement. En effet, ce groupe s'individualise principalement par le poids de la variable «surface du bassin-versant », qui est très dépendante du degré d'évolution de la forme. Les formes les plus récentes et/ou les moins retouchées par l'érosion pourraient être associées à un score factoriel élevé sur l'axe F2.

Ce travail n'avait pas pour objectif initial d'établir une relation stricte entre morphométrie et origine des formes mais, avant tout, de mettre en évidence la diversité morphologique des formes regroupées sous le vocable unique de dépression fermée. L'impossibilité de prendre en compte les formes les plus petites dans une analyse quantitative à partir des données existantes constitue la principale limite de ce travail. Dans le département des Pyrénées Orientales, où un MNT LIDAR à résolution spatiale métrique est disponible, la prise en compte de l'ensemble des formes est envisageable. La disponibilité annoncée à l'échelle nationale d'un MNT à résolution métrique dans le cadre du Référentiel à Grande Echelle de l'IGNF pourrait permettre d'étendre cette démarche à l'ensemble de la zone étudiée, voire d'y intégrer les dépressions du piémont de la Montagne Noire. L'inventaire des formes se trouvera probablement accru par cette démarche.

La mise à disposition d'un MNT à haute ou très haute résolution permettra de mieux prendre en compte les paramètres de formes en incluant notamment des indicateurs de la forme des versants et une discrimination plus pertinente des formes des bassins versants associés aux dépressions. Ces paramètres sont d'une première importance afin de prendre en considération les formes non plus dans une dimension statique mais dynamique. Elles pourraient permettre de mieux discriminer au sein du groupe g3 des sous-groupes relevant de processus identiques mais ayant connu des évolutions divergentes. Cette approche, couplée avec une prise en compte du début de la sédimentation dans les dépressions par généralisation des carottages, pourrait permettre de mieux cerner le rôle de l'évolution postglaciaire dans la morphologie actuelle de ces dépressions fermées. L'analyse géochimique des sédiments, notamment l'étude géochimique des dépôts halins, permettra probablement de trancher définitivement l'origine halocinétique des grandes formes du groupe $\mathrm{g} 3$.

\section{Conclusions}

Conformément aux objectifs prédéfinis, ce travail a mis en évidence le caractère fréquent des formes de type dépressions fermées dans les paysages du Languedoc central et occidental ainsi que du Roussillon, d'une part, et la diversité morphologique de ces formes, d'autre part.

Le croisement d'indicateurs géologiques, géomorphologiques et typonymiques/historiques s'est avéré une bonne méthode pour inventorier ces formes malgré leur diversité. Les descripteurs utilisés, relatifs à la forme en plan et à la forme en coupe, se sont avérés de bons discriminants et ont permis de dégager trois grands types de forme. Si les formes du groupe g1 sont exclusivement présentes en Languedoc central, les formes des groupes g2 et g3 sont plus ubiquistes. Cette typologie géomorphométrique, donc statique, permet toutefois d'émettre une hypothèse sur des origines multiples et non unique des processus géomorphologiques responsables du façonnement des formes et de leur évolution. Si la plupart des formes des groupes g1 et g2 pourraient effectivement être pro parte le résultat de processus hydro-éoliens avec lesquels ces formes sont compatibles, la mise en relation des dépressions du groupe g3 avec ce processus semble plus douteuse. Une origine diapirique pourrait être envisagée pour ce groupe, ainsi que pour certains éléments du groupe g1. L'érosion postérieure à la formation des dépressions pourrait avoir conduit à faire diverger, d'un point de vue géomorphologique, des formes initialement proches. Ce processus ne pourra être approché que par des travaux de cartographie de terrain à très grande échelle.

La cartographie géomorphologique systématique de ces dépressions ainsi que l'étude de leur relation avec les niveaux de terrasse alluviale d'une part et la comparaison avec les données géomorphométriques des dépressions du pays d'Istres d'autre part devraient permettre d'affiner les groupes, leur attribution à des processus morphogéniques et la chronologie de la formation de ces modelés.

\section{Remerciements}

Nous remercions les trois relecteurs qui, par leurs remarques et leurs suggestions, nous ont permis d'améliorer le contenu de ce texte.

\section{Références}

Abbé J.-L. (2005) - L'eau et la terre : Les transformations du milieu humide en Languedoc méditerranéen au Moyen Age. $21^{\mathrm{e}}$ colloque des archives d'Agde. Association pour la Promotion des Archives d'Agde et de sa Région, Agde, 16 p. (http:// halshs.archives-ouvertes.fr/docs/00/13/95/11/PDF/Texte_article_Agde.pdf).

Abbé J.-L. (2006) - A la conquête des étangs. L'aménagement de l'espace en Languedoc méditerranéen (XIIe-XVe siècle), P. U. du Mirail, Toulouse, 2006, coll. Tempus, $331 \mathrm{p}$.

Abbé J.-L. (2009) - Autour de l'étang de Montady. Espace, environnement et mise en valeur du milieu humide en Languedoc, des oppida à nos jours. Rapport de Programme Collectif de Recherche, SRA Languedoc-Roussillon, $182 \mathrm{p}$.

Alsop G.I., Archer A., Hartley A.J., Grant N. (2012) - Salt tectonic, sediments and prospectivity. Geological Society London, London, $624 \mathrm{p}$.

Ambert P. (1973) - Géomorphologie de l'étang de Berre et de ses bordures. Thèse de $3^{\mathrm{e}}$ cycle, université de Provence (Aix-Marseille 1), $465 \mathrm{p}$.

Ambert P. (1994) - L'évolution géomorphologique du Languedoc Central depuis le Néogène (Grands Causses méridionaux-Piémont languedocien). Bureau de Recherches Géologiques et Minières, Orléans, France, 210 p. 
Ambert P., Clauzon G. (1992) - Morphogénèse éolienne en ambiance périglaciaire : les dépressions fermées du pourtour du Golfe du Lion (France méditerranéenne). Zeitschrift für Geomorphologie Suppl. 84, 55-71.

Arnal H. (1971) - Phénomènes périglaciaires dans la basse vallée $d u$ Rhône : formations quaternaires du rebord sud et alluvions du fond de l'étang de Pujaut. Bulletin de l'Association Française pour l'Étude du Quaternaire, 8,3, 145-149.

Azemar J., Carriere P. (1980) - L'aménagement hydraulique de la dépression de Villeneuve-de-la-Raho (Pyrénées-Orientales). Bulletin de la Société Languedocienne de Géographie, 14, 2-3, 249-265.

Barrot J. (1975) - Carte géomorphologique détaillée de la France au 1:50000 - feuille Narbonne.

Bécat J. (1977) - Atlas de Catalunya. Terra Nostra, Prades, 2 volumes, 138 planches, $212 \mathrm{p}$.

Brun J.-P., Fort X. (2008) - Entre sel et terre, Structures et mécanismes de la tectonique salifère. Société géologique de France, Vuibert, Paris, $154 \mathrm{p}$.

Calvet M. (1996) - Morphogenèse d'une montagne méditerranéenne : les Pyrénées orientales. Thèse d'État, Document du BRGM n $^{\circ} 255,3$ t, 1177 p.

Carozza J.-M., Puig C., Mazier F., Valette P., Odiot T. (sous presse) - Dépressions fermées, étangs et zones humides continentales de la plaine du Roussillon : caractérisation, typologie et trajectoires d'évolution (Protohistoire, période Moderne). In Trément F., Franceschelli C. (Eds.) Aménagement et exploitation des zones humides depuis l'Antiquité. Presses universitaires de Clermont-Ferrand, Collection « Histoire de l'environnement », Clermont-Ferrand.

Carriere P. (1980) - Le dessèchement et l'aménagement hydraulique de l'étang de Montady (Hérault). Bulletin de la Société Languedocienne de Géographie, 14, 2-3, 199-214.

Caucanas S. (1995) - Assèchements en Roussillon (XII ${ }^{\mathrm{e}}$ $\mathrm{XIV}^{\mathrm{e}}$ siècles). In Senac P. (Ed.) Histoire et archéologie des terres catalanes au Moyen-Age. Presses universitaires de Perpignan, Perpignan, 269-278.

CENLR [Conservatoire des Espaces Naturels du Languedoc Roussillon] (2000) - Inventaire et cartographie des étangs asséchés du Languedoc Roussillon. Rapport inédit, 28 p.

Chabert F. (1980) - L'étang de Marseillette (Aude) : Historique de son assèchement et mise en valeur actuelle. Bulletin de la Société Languedocienne de Géographie, 14, 2-3, 230-247.

Collina-Girard J. (1989) - Application des techniques de l'analyse cartographique (analyse morphostructurale) : l'exemple du Roussillon (Pyrénées-Orientales, France). Géologie Méditerranéenne, XVI, 4, 321-340.

Degeai J.-P. (2004) - Mesure de l'érosion post-éruptive autour des cratères de maars en inversion de relief dans le Massif central français. Géomorphologie : relief, processus, environnement 4, 285-304.

Derruau M. (1996) - Géomorphologie et histoire de l'étang de Capestang. Bulletin de la Société Languedocienne de Géographie, 3-4, 221-253.

French H.M., Demitroff M. (2001) - Cold-Climate Origin of the Enclosed Depressions and Wetlands ('Spungs') of the Pine Barrens, Southern New Jersey, USA. Permafrost and Periglacial Processes 12, 337-350.
Gaullier V. (1993) - Diapirisme salifère et dynamique sédimentaire dans le bassin liguro-provençal : données sismiques et modèles analogiques. Thèse de doctorat, université Pierre et Marie Curie (Paris 6), $327 \mathrm{p}$.

Gorini C. (1993) - Géodynamique d'une marge passive : le Golfe du Lion (Méditerranée occidentale). Thèse, université Toulouse 3, $267 \mathrm{p}$.

Gottis M. (1968) - Les dépressions fermées du Languedoc : Hypothèses sur leur mode de formation. Actes de la Société Linéenne de Bordeaux, 105, 1-5.

Goudie A.S., Wells G.L. (1995) - The nature, distribution and formation of pans in arid zones. Earth Science Reviews 38, 169.

Guilaine J. (Ed.) (1993) - Temps et espaces dans le bassin de l'Aude du Néolithique à l'âge du Fer. Centre d'anthropologie des sociétés rurales, Toulouse, $442 \mathrm{p}$.

Jalut G., Dedoubat J.-J., Fontugne M., Otto T. (2009) - Holocene circum-Mediterranean vegetation changes: Climate forcing and human impact. Quaternary International 200, 4-18.

Kranjc A. (2013) - Classification of closed depressions in carbonate karst. In Shroder J., Frumkin A. (Eds.) Treatise on Geomorphology. Academic Press, San Diego, CA, 6, Karst Geomorphology, 104-111.

Le Cann C. (1987) - Le diapirisme dans le bassin liguro-provençal (Méditerranée occidentale) : Relations avec la sédimentation et la tectonique; conséquences geodynamiques. Thèse de doctorat, université de Bretagne occidentale, 299 p.

Lespinasse P. (1982) - Notice explicative de la feuille Narbonne à 1/50 000. BRGM, Orléans, 48 p.

Martzluff M. (1993) - Villeneuve-de-la-Raho : un terroir en Roussillon, géologie, archéologie, histoire. Le publicateur, Perpignan, $96 \mathrm{p}$.

Nicod J. (1991) - Phénomènes karstiques dans le Trias du département du Var. URA $903 d u$ CNRS, Travaux nXX, 5-14.

Norton O.R. (1998) - Rocks from space: meteorites and meteorite hunters. Mountain Press Publications, Missoula, 447 p.

Pissart A. (1987) - Géomorphologie périglaciaire. Laboratoire de géomorphologie et de géologie du Quaternaire de l'université de Liège, Liège, $133 \mathrm{p}$.

Puig C. (2003) - Les campagnes roussillonnaises au Moyen Âge : pratiques agricoles et paysagères entre le $12^{e}$ et la première moitié $d u 14^{e} s$. Thèse, université Toulouse 2,918 p.

Roche M. (1963) - Hydrologie de surface. Gauthier-Villars, Paris, $429 \mathrm{p}$.

Sanchez J.A., Perez A., Coloma P., Martinez-Gil J. (1997) Combined effects of groundwater and aeolian processes in the formation of the northernmost closed saline depressions of Europe: north-east Spain. Hydrological Processes 12, 813820.

Sears D.W. (1978) - The nature and origin of meteorites. Oxford University Press, Monographs on Astronomical Subjects 5, $195 \mathrm{p}$.

Seranne M. (1999) - The Gulf of Lion continental margin (NW Mediterranean) revisited by IBS: an overview. In Durand B., Jolivet L., Horváth F., Seranne M. (Eds.) The Mediterranean Basins: Tertiary Extension within the Alpine Orogen. The Geological Society, London, 15-36. 
Seranne M., Benedicto A., Labaum P. (1995) - Structural style and evolution of the Gulf of Lion Oligo-Miocene rifting: Role of the Pyrenean orogeny. Marine and Petroleum Geology 12-8, 809-820.

Van Vliet-Lanoë B. (2005) - La planète des glaces, histoire et environnements de notre ère glaciaire. Vuibert, Paris, $470 \mathrm{p}$.

Wanniez P. (2008) - Cartographie thématique et analyse des données avec Philcarto 5xx pour Windows. Doc de GRANIT 1, Université Bordeaux 2, Bordeaux, 105 p.
White W.B., White E.L. (2006) - Size scales for closed depression landforms: the place of tiankengs. Speleogenesis and Evolution of Karst Aquifers 4, 1, 1-1

Yechieli Y., Wood W. (2002) - Hydrogeologic processes in saline systems: playas, sabkhas, and saline lakes. Earth Sciences Reviews 58, 343-365.

Article soumis le 27 février 2013, accepté le 13 mai 2013. 